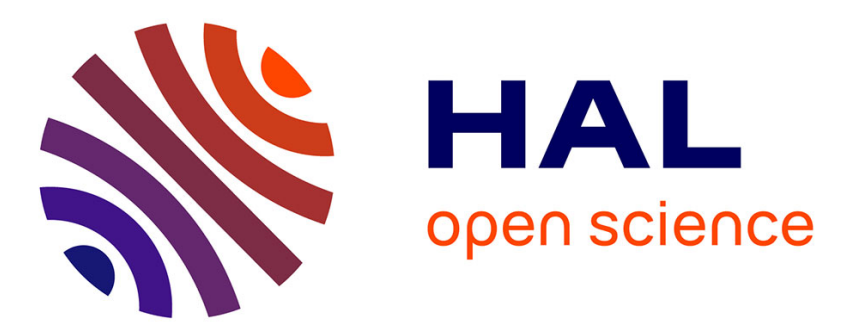

\title{
Robust Bayesian super-resolution approach via sparsity enforcing a priori for near-field aeroacoustic source imaging
}

\author{
Ning Chu, Ali Mohammad-Djafari, José Picheral
}

\section{- To cite this version:}

Ning Chu, Ali Mohammad-Djafari, José Picheral. Robust Bayesian super-resolution approach via sparsity enforcing a priori for near-field aeroacoustic source imaging. Journal of Sound and Vibration, 2013, 332 (18), pp.4369-4389. hal-00794230v2

\section{HAL Id: hal-00794230 \\ https://hal.science/hal-00794230v2}

Submitted on 16 Apr 2013

HAL is a multi-disciplinary open access archive for the deposit and dissemination of scientific research documents, whether they are published or not. The documents may come from teaching and research institutions in France or abroad, or from public or private research centers.
L'archive ouverte pluridisciplinaire HAL, est destinée au dépôt et à la diffusion de documents scientifiques de niveau recherche, publiés ou non, émanant des établissements d'enseignement et de recherche français ou étrangers, des laboratoires publics ou privés. 


\title{
Robust Bayesian super-resolution approach via sparsity enforcing a priori for near-field aeroacoustic source imaging
}

\author{
Ning CHU $^{\mathrm{a}, 1, *}$, Ali MOHAMMAD-DJAFARI ${ }^{\mathrm{a}}$, José PICHERAL ${ }^{\mathrm{b}}$ \\ ${ }^{a}$ Laboratoire des signaux et systèmes (L2S), CNRS-SUPELEC-UNIV PARIS SUD, 91192 GIF-SUR-YVETTE, FRANCE \\ ${ }^{b}$ SUPELEC, Département du Signal et Systèmes Electroniques, 91192 GIF-SUR-YVETTE, FRANCE
}

\begin{abstract}
Near-field aeroacoustic imaging has been the focus of great attentions of researchers and engineers in aeroacoustic source localization and power estimation for decades. Recently the deconvolution and regularization methods have greatly improved spatial resolution of the beamforming methods. But neither are they robust to background noises in the low Signal-to-Noise Ratio (SNR) situation, nor do they provide a wide dynamic range of power estimation.

In this paper, we first propose an improved forward model of aeroacoustic power propagation, in which, we consider background noises and forward model uncertainty for the robustness. To solve the inverse problem, we then propose a robust Bayesian super-resolution approach via sparsity enforcing a priori. The sparse prior of source powers can be modeled by double exponential distribution, which can improve the spatial resolution and promote wide dynamic range of source powers. Both the hyperparameters and source powers can be alternatively estimated by the Bayesian inference approach based on the joint Maximum A Priori optimization. Finally our Bayesian approach is compared with some of the state-of-the-art methods on simulated, real and hybrid data. The main advantages of our approach are of robustness to noise, a wide dynamic range, super spatial resolution, and non-necessity for prior knowledge of the source number or SNR. It is feasible to apply it for aeroacoustic imaging with the 2D non-uniform microphone array in wind tunnel tests, especially for near-field monopole and extended source imaging.
\end{abstract}

Keywords: Source localization, Bayesian inference, parameter estimation, aeroacoustic imaging, sparsity, super-resolution

\section{Introduction}

Aeroacoustic imaging is a standard technique for mapping the positions and powers of aeroacoustic sources with microphone arrays, which provides the insight into the mechanisms and properties of aeroacoustic sources. Nowadays, near-field aeroacoustic imaging with non-uniform arrays (NUA) has been widely studied and applied in various applications for stationary, moving and rotating objects: machinery manufacturing in wind tunnel tests, aeroacoustic comfort in transportation, performance of stator and rotor in wind power etc. [1-4]. Based on the physical mechanism and mathematical tool, classical imaging methods could be loosely classified as: time-reversal aeroacoustic imaging [5], Near-field Acoustic Holography (NAH) [6], beamforming [7] and inverse problems [8].

In this paper, we focus on the beamforming and inverse problem. The beamforming method [7] is direct, simple and fast, but its spatial resolution and dynamic range are limited due to the high sidelobes and spatial aliasing effects, especially at low frequency. Though MUltiple SIgnal Classification (MUSIC) [9] greatly improves the beamforming resolution, it still requires high SNR and the exact number of sources, moreover MUSIC could not directly estimate

\footnotetext{
${ }^{\text {ये }}$ Paper partly based on that accepted by 2012IEEE International Conference on Image Processing (ICIP), pp2529-2532 Sep.30-Oct.03, 2012, Orlando, USA.

*Principal corresponding author: Ning.CHU@1ss.supelec.fr (Ning CHU). Tel. : +33 (0)1 6985 1743. Fax : 0033 (0)1 69851765

Email addresses: djafari@lss.supelec.fr (Ali MOHAMMAD-DJAFARI), jose.picheral@supelec.fr (José PICHERAL)

${ }^{1}$ The author's PhD study is financed by China Scholarship Council (CSC) and École Supérieure d'Électricité (SUPELEC) FRANCE
} 


\begin{tabular}{|c|c|c|c|}
\hline Nomenclature & & $\mu$ & gradient step \\
\hline $\mathbf{a}_{n} \in \mathbb{C}^{M}$ & steering vector for $\mathbf{p}_{n}$ & $\rho$ & reflecting coefficient \\
\hline $\mathbf{a}_{k}^{*} \in \mathbb{C}^{K}$ & steering vector for $\mathbf{p}_{k}^{*}$ & $\sigma^{2}$ & background noise variance \\
\hline$\tilde{\mathbf{a}}_{n} \in \mathbb{C}^{M}$ & beamforming vector for $\mathbf{p}_{n}$ & $\tau_{n, m}$ & time for distance $r_{n, m}$ \\
\hline $\mathbf{A} \in \mathbb{C}^{M \times N}$ & steering matrix for $\mathbf{P}$ & $\tau_{-n, m}$ & time for distance $r_{-n, m}$ \\
\hline $\mathbf{A}^{*} \in \mathbb{C}^{M \times K}$ & steering matrix for $\mathbf{P}^{*}$ & $\theta$ & hyperparameters to be estimated \\
\hline$c_{n, q}$ & impulse response of sensor array, $c_{n, q} \in \mathbf{C}$ & $\theta_{0}$ & known parameters \\
\hline $\mathbb{C}$ & complex number domain & $\theta_{1}$ & hyperparameters in forward model \\
\hline $\mathbf{C} \in \mathbb{R}^{N \times N}$ & power propagation matrix & $\theta_{2}$ & hyperparameters in prior models \\
\hline $\mathrm{d}$ & aperture of sensor array & $\xi \in \mathbb{R}^{N}$ & forward model uncertainty \\
\hline $\mathrm{D}$ & distance from array to source plane & $\Delta B$ & beamforming spatial resolution \\
\hline$e_{m}$ & noise at sensor $\mathrm{m}$ & $\Delta p$ & scanning step \\
\hline $\mathbf{e} \in \mathbb{C}^{M}$ & background noises at $\mathrm{M}$ sensors & $\overline{\Delta x^{*}}$ & averaged estimation error of $\mathbf{x}^{*}$ \\
\hline$f_{l}$ & frequency at $l$ th bin & $1_{N} \in \mathbb{R}^{N}$ & vector with $\mathrm{N}$ elements of 1 \\
\hline I & number of sampling block & & \\
\hline $\mathbf{I}_{M} \in \mathbb{R}^{M \times M}$ & identity matrix with size $\mathrm{M} \times \mathrm{M}$ & Operator & \\
\hline $\mathrm{K}$ & number of modeled sources & $\mathrm{e}$ & natural exponential \\
\hline $\mathrm{L}$ & number of frequency bins & $\exp \{\}$ & natural exponential \\
\hline M & number of microphone sensors & $\mathbb{E}\{\cdot\}$ & mathematical expectation \\
\hline $\mathrm{N}$ & number of scanning points & $\operatorname{diag}[\cdot]$ & diagonal values of matrix \\
\hline $\mathbf{p}_{n} \in \mathbb{R}^{3}$ & coordinates of source $n$ & $\ln \{\cdot\}$ & natural log \\
\hline $\mathbf{P} \in \mathbb{R}^{N \times 3}$ & coordinate matrix of $\mathrm{N}$ sources & $\operatorname{tr}\{\cdot\}$ & trace of matrix \\
\hline$\overline{\mathbf{p}}_{m} \in \mathbb{R}^{3}$ & coordinates of sensor $\mathrm{m}$ & $\nabla(\cdot)$ & gradient \\
\hline$\overline{\mathbf{P}} \in \mathbb{R}^{M \times 3}$ & coordinate matrix of $\mathrm{M}$ sensors & $\|\cdot\|$ & spectral norm of square matrix \\
\hline $\mathbf{p}_{k}^{*} \in \mathbb{R}^{3}$ & coordinates of modeled source $\mathrm{k}$ & $\|\cdot\|_{l}$ & $l$ norm of a vector \\
\hline $\mathbf{P}^{*} \in \mathbb{R}^{K \times 3}$ & coordinate matrix of $\mathrm{K}$ modeled sources & $(\cdot)^{T}$ & transpose \\
\hline$r_{n, m}$ & distance from source $\mathrm{n}$ to sensor $\mathrm{m}$ & $(\cdot)^{H}$ & conjugate transpose \\
\hline$r_{-n, m}$ & distance from source $-n$ to sensor $m$ & $(\cdot)^{(k)}$ & $k$ th iteration \\
\hline $\mathbb{R}$ & real number domain & $*$ & convolution \\
\hline $\mathbf{R} \in \mathbb{C}^{M \times M}$ & measured cross spectrum matrix & & \\
\hline$s_{n}$ & signal of source $n$ & Abbreviation & \\
\hline $\mathbf{s} \in \mathbb{C}^{N}$ & signal vector of $\mathrm{N}$ sources & AGWN & Additive Gaussian White Noise \\
\hline & signal of modeled source $\mathrm{k}$ & $\mathrm{CMF}$ & Covariance Matrix Fitting method \\
\hline $\mathbf{s}^{*} \in \mathbb{C}^{K}$ & signal vector of $\mathrm{K}$ modeled sources & $\mathrm{dB}$ & decibel \\
\hline $\mathrm{T}$ & total number of samplings & DAMAS & Deconvolution Approach for Mapping \\
\hline $\mathrm{v}$ & wind speed & & of Acoustic Source \\
\hline$x_{n}$ & power of source $n$ & $\mathcal{D E}$ & Double Exponential \\
\hline$x_{k}^{*}$ & power of modeled source $\mathrm{k}$ & DFT & Discrete Fourier Transform \\
\hline $\mathbf{x} \in \mathbb{R}^{N}$ & power vector of $\mathrm{N}$ sources & DR & Diagonal Removal \\
\hline $\mathbf{x}^{*} \in \mathbb{R}^{K}$ & power vector of $\mathrm{K}$ modeled sources & $\mathcal{G G}$ & Generalized Gaussian \\
\hline $\mathbf{X} \in \mathbb{R}^{N \times N}$ & cross spectrum matrix of $\mathrm{N}$ sources & i.i.d & independent and identically distributed \\
\hline $\mathbf{y} \in \mathbb{R}^{N}$ & modeled power vector of $\mathrm{N}$ sources & MAP & Maximum A Posteriori \\
\hline$\tilde{\mathbf{y}} \in \mathbb{R}^{N}$ & beamforming power vector of $\mathrm{N}$ sources & PDF & Probability Density Function \\
\hline $\begin{array}{l}z_{i, m} \\
z_{i} \in \mathbb{C}^{M}\end{array}$ & $\begin{array}{l}\text { measurements in } i \text { th block at sensor } \mathrm{m} \\
\text { measurements } i \text { th block at } \mathrm{M} \text { sensors }\end{array}$ & PSF & Point Spread Function \\
\hline$\alpha$ & regularization parameter & Subscripts & \\
\hline$\beta$ & shape parameter in $\mathcal{D} \mathcal{E}(x)$ model & $m, n$ & term associated with $m$ and $n$ \\
\hline$\delta_{i}$ & relative error of image reconstruction & $-\mathrm{n}$ & mirror source $n$ \\
\hline$\gamma$ & scale parameter in $\mathcal{D E}(x)$ model & $\mathrm{n}^{\prime}$ & equivalent source $n$ \\
\hline$\lambda$ & wavelength & $w b$ & wide-band \\
\hline
\end{tabular}


source powers. Many state-of-the-art methods have been explored based on beamforming and MUSIC, such as the Orthogonal Beamforming [10] and Near-Field Focalization (NFF) [11].

The inverse problem consists of using the measurements of forward physical model to estimate parameters that characterize this forward model [8]. The deconvolution method is one of the commonly used techniques. For aeroacoustic imaging, the CLEAN [12] and RELAX methods [13] can iteratively extract strong sources from a fuzzy beamforming image. However, they tend to eliminate weak sources drowned in the background noises; besides, some important parameters (attenuation factor, iteration number) have to be selected empirically. Recently, the Deconvolution Approach for Mapping of Acoustic Source (DAMAS) method [14] has been a breakthrough and effectively applied in wind tunnel tests by NASA. DAMAS gives an iterative solution of a determined system of linear equation under non-negative constraint, but DAMAS is sensitive to background noises and suffers from slow convergence. DAMAS2 and DAMAS3 [15] accelerate the DAMAS by confining the Point Spread Function (PSF) of sensor array to be shift invariant, but this assumption inevitably affects spatial resolution. To overcome the multiple solutions of deconvolution methods, one needs to add regularization constraints or prior information on the unknown variables. For example, DAMAS with sparsity constraint (SC-DAMAS) [16] greatly improves the spatial resolution, but it is still not robust to noise interference. The Covariance Matrix Fitting (CMF) method [17] can estimate the noise power and improve the robustness of SC-DAMAS, but it is not feasible to use for high resolution imaging, since CMF has huge dimensionality of variables in covariance matrix. The $\ell_{1}$ norm is used for enforcing sparsity [18, 19]. And paper [20] proposed to use the $\ell_{1}$ norm with Iteratively Re-weighted Least Square methods for coherent/incoherent, distributed/multipole sources imaging. However, some of $\ell_{1}$ methods have to know source number beforehand, or make necessary approximation. Recently, the authors have proposed the Robust DAMAS with Sparse Constraint (SC-RDAMAS) [21], which achieves higher resolution and background noise estimation, but the parameter of sparsity constraint still depends on source number estimation. Recently, the Bayesian framework has been successfully applied in acoustic imaging $[22,23]$ and overcome the drawbacks of deconvolution and regularization methods.

To summarize, all the above methods have excellent performances on some focused aspects, but there is no onefits-all methods, and most of them suffer one of the following drawbacks: poor spatial resolution, sensitivity to background noises, narrow dynamic range and high computational cost. In addition, most of them need to set some important parameters that must be tuned accurately for each case to obtain good performance.

In this paper, our main motivation is to investigate a robust approach with high spatial resolution and wide dynamic range. In order to overcome most of the drawbacks of existing methods, we propose a robust Bayesian super-resolution approach via sparsity enforcing a priori. In proposed approach, we reconstruct source powers and positions from strong noise interference, and jointly estimate the hyperparameters of the forward and prior models.

The novelties in this paper are 1) to improve the robustness of forward model of aeroacoustic power propagation, we take account for the background noises and forward model uncertainty (the unpredictable parts in aeroacoustic propagation); 2) to obtain the robust and high resolution solutions for the improved forward model, proposed Bayesian inference approach applies the Double Exponential $(\mathcal{D E})$ prior model to enforce the sparsity of source power distribution, and promote large dynamic range of estimated powers; 3 ) instead of empirically tuning the parameters, proposed approach automatically rebuilds source positions and powers, and alternatively estimates hyperparameters, such as the variance of background noises, the power of forward model uncertainty and other parameters in prior model.

The advantages of the proposed Bayesian approach are super spatial resolution, robustness to background noises, wide dynamic range of power estimations and the non-necessity of priors like source number or SNR. It can be applied for the near-field monopole and extended aeroacoustic source imaging on the surface of the static object in wind tunnel test based on the 2D NUA array.

This paper is organized as follows: Section 2 briefly introduces the forward model of near-field aeroacoustic signal propagation. In Section 3, we present classical beamforming and deconvolution methods for aeroacoustic imaging. Then we improve the forward model of aeroacoustic power propagation in Section 4. And our proposed Bayesian inference approach is presented in Section 5. On simulations, Section 6 demonstrates performance comparisons of the proposed approach with state-of-the-art methods for the monopole and extended source imaging. Results of the real data in wind tunnel experiments are illustrated in Section 7. In order to further prove the effectiveness of proposed Bayesian approach, Section 8 demonstrates its performances on hybrid data, in which some known synthetic sources are added to the real data. Finally the conclusions and perspectives of the paper are summarized in Section 9. 


\section{Forward model of aeroacoustic signal propagation in the near-field}
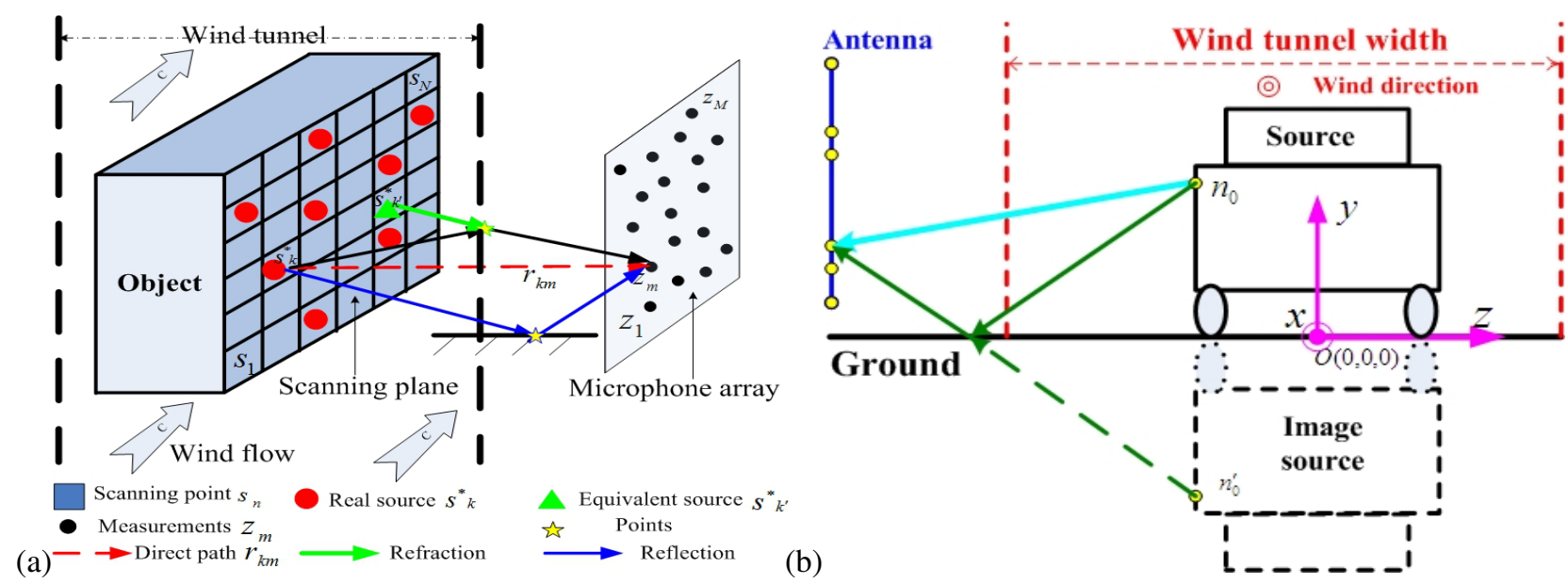

Figure 1: Illustration of open wind tunnel tests: (a) wind refraction effect and (b) ground reflection effect.

For aeroacoustic imaging, a source is supposed to be a monopole source, since this assumption is widely used by many researchers $[10,12-14,16]$. An extended source can be modeled by several uncorrelated monopole sources, and extended sources are used to represent the sources with different directivity patterns as discussed in [14, 24]. In this paper, we suppose the monopole model in order to simplify the physical process and explicitly build up the forward model of aeroacoustic signal propagation. In order to fit the physical source, we explore the extended source imaging in simulated and hybrid data.

\subsection{Assumptions}

Before modeling, we make three necessary assumptions on sources, background noises and sensors:

- Aeroacoustic sources are spatially punctual and uncorrelated monopoles, and they locate on the same plane;

- Background noises are the Additive Gaussian White Noise (AGWN) with variance $\sigma^{2}$, mutually independent and identically distributed (i.i.d), and also independent to aeroacoustic sources;

- Microphone sensors are omnidirectional with unitary gain.

- Reverberations could be negligible in the anechoic wind tunnel.

\subsection{Forward model of aeroacoustic signal propagation}

Figure 1 illustrates near-field aeroacoustic imaging based on 2D NUA array in the open wind tunnel, where sensor array locates outside the wind flow. We consider $\mathrm{M}$ sensors and $\mathrm{K}$ near-field source signals $\mathbf{s}^{*}$ at positions $\mathbf{P}^{*}=\left[\mathbf{p}_{1}^{*}, \cdots, \mathbf{p}_{K}^{*}\right]^{T}$ with $\mathbf{p}_{k}^{*}$ being $3 \mathrm{D}$ coordinate of $k$ th modeled source. For each microphone sensor, aeroacoustic signals are sampled, then divided into I blocks with L samplings in each block; thus the total samplings are $T=I L$. Since the discrete signals are usually wide-band, we apply the Discrete Fourier Transform (DFT) in time domain of each block in order to obtain L narrow frequency bins. Therefore the measured signals $\mathbf{z}_{i}\left(f_{l}\right)=\left[z_{i, 1}\left(f_{l}\right), \cdots, z_{i, M}\left(f_{l}\right)\right]^{T}$ at frequency $f_{l}(l \in[1, \mathrm{~L}])$ in the sampling block $\mathrm{i}(i \in[1, I])$ can be modeled as $[13]$ :

$$
\mathbf{z}_{i}\left(f_{l}\right)=\mathbf{A}^{*}\left(\mathbf{P}^{*}, f_{l}\right) \mathbf{s}_{i}^{*}\left(f_{l}\right)+\mathbf{e}_{i}\left(f_{l}\right),
$$

where $\mathbf{e}_{i}\left(f_{l}\right)=\left[e_{i, 1}\left(f_{l}\right), \cdots, e_{i, M}\left(f_{l}\right)\right]^{T}, \mathbf{e}_{i}\left(f_{l}\right) \in \mathbb{C}^{M}$ denotes i.i.d AGWN noise; $\mathbf{s}_{i}^{*}\left(f_{l}\right)=\left[s_{i, 1}^{*}\left(f_{l}\right), \cdots, s_{i, K}^{*}\left(f_{l}\right)\right]^{T}, \mathbf{s}_{i}^{*}\left(f_{l}\right) \in$ $\mathbb{C}^{K}$ denotes the DFT of the source signals, $\mathbf{A}^{*}\left(\mathbf{P}^{*}, f_{l}\right)=\left[\mathbf{a}^{*}\left(\mathbf{p}_{1}^{*}, f_{l}\right), \cdots, \mathbf{a}^{*}\left(\mathbf{p}_{K}^{*}, f_{l}\right)\right], \mathbf{A}^{*}\left(\mathbf{P}^{*}, f_{l}\right) \in \mathbb{C}^{M \times K}$ denotes the near-field steering matrix. Therefore we get the forward model of aeroacoustic signal propagation in Eq.(1). 
Unlike [13], we account for the ground reflection effect as shown in Fig.1b, since the object vehicle is near to the ground, the ground reflection can not be negligible in wind tunnel experiments. Therefore steering vector $\mathbf{a}^{*}\left(\mathbf{p}_{k}^{*}, f_{l}\right)$ is composed by the direct propagation vector $\mathbf{a}_{d}^{*}\left(\mathbf{p}_{k}^{*}, f_{l}\right)$ and the ground reflection vector $\mathbf{a}_{r}^{*}\left(\mathbf{p}_{-k}^{*}, f_{l}\right)$ :

$$
\mathbf{a}^{*}\left(\mathbf{p}_{k}^{*}, f_{l}\right)=\mathbf{a}_{d}^{*}\left(\mathbf{p}_{k}^{*}, f_{l}\right)+\rho \mathbf{a}_{r}^{*}\left(\mathbf{p}_{-k}^{*}, f_{l}\right),
$$

where $\rho$ denotes the reflecting coefficient $(0 \leq \rho \leq 1)$, whose value mainly depends on ground conditions (material, temperature, humidity, etc.). For the real data used in this paper, $\rho=0.8$ is used in wind tunnel S2A experiments [25], thanks to the contribution of researchers in Renault Lab.

In Eq.(2), the direct propagation vector $\mathbf{a}_{d}^{*}\left(\mathbf{p}_{k}^{*}, f_{l}\right)$ is defined as:

$$
\mathbf{a}_{d}^{*}\left(\mathbf{p}_{k}^{*}, f_{l}\right)=\left\{\frac{1}{r_{k, 1}} \exp \left\{-j 2 \pi f_{l} \tau_{k, 1}\right\}, \cdots, \frac{1}{r_{k, m}} \exp \left\{-j 2 \pi f_{l} \tau_{k, m}\right\}, \cdots, \frac{1}{r_{k, M}} \exp \left\{-j 2 \pi f_{l} \tau_{k, M}\right\}\right\}^{T},
$$

where $\tau_{k, m}$ is the propagation time from $s_{k}^{*}$ to sensor $m$, and $r_{k, m}$ is the propagation distance during $\tau_{k, m}$.

Moreover, in Eq.(2), the ground reflection vector $\mathbf{a}_{r}^{*}\left(\mathbf{p}_{-k}^{*}, f_{l}\right)$ is defined as:

$$
\mathbf{a}_{r}^{*}\left(\mathbf{p}_{-k}^{*}, f_{l}\right)=\left\{\frac{1}{r_{-k, 1}} \exp \left\{-j 2 \pi f_{l} \tau_{-k, 1}\right\}, \cdots, \frac{1}{r_{-k, m}} \exp \left\{-j 2 \pi f_{l} \tau_{-k, m}\right\}, \cdots, \frac{1}{r_{-k, M}} \exp \left\{-j 2 \pi f_{l} \tau_{-k, M}\right\}\right\}^{T},
$$

where $\mathbf{p}_{-k}^{*}$ denotes mirror positions of $s_{k}^{*}$, thus $\mathbf{p}_{-k}^{*}$ and $\mathbf{p}_{k}^{*}$ are symmetric to the ground.

Since the sensor array locates outside the wind flow, the medium for aeroacoustic propagation is not uniform, thus the refraction will happen on the interface between the common air and wind flow. In Appendix C and Appendix D, the actual $r_{k, m}, r_{-k, m}$ and $\tau_{k, m}, \tau_{-k, m}$ are formulated in details: we apply the concepts of equivalent sources and mirror sources to deal with the wind refraction and ground reflection respectively.

\section{Classical inverse solutions}

In Eq.(1), since source positions $\mathbf{P}^{*}$ and source signals $\mathbf{s}^{*}$ are both unknown, it is thus a nonlinear system of equations. In order to transform Eq.(1) into a linear system, the classical inverse problem is based on the discretization of the source plane, as illustrated in Fig.1a. The scanning plane is equally discretized into $\mathrm{N}$ scanning points at positions $\mathbf{P}$, satisfying $\mathrm{N}>\mathrm{M}>\mathrm{K}$ and $\mathbf{P}^{*} \subset \mathbf{P}$. These scanning points are thus regarded as the potential source signals $\mathbf{S}$ at positions $\mathbf{P}$. in the following, the term source $\mathrm{n}$ means the source on the position $\mathbf{p}_{n}$. Therefore source positions $\mathbf{P}$ are known after discretizing the source plane, and $\mathbf{P}^{*}$ can be estimated as the positions, where the corresponding source signals are non-trivial values. The smaller grid is discretized, the higher spatial resolution can be achieved. Each scanning point $\mathbf{s}_{n}$ could be regarded as a potential source signal, then in the space domain we have

$$
\left\{\begin{array}{lll}
s_{n}=s_{k}^{*} & \text { for } & \mathbf{p}_{n}=\mathbf{p}_{k}^{*} \\
s_{n}=0 & \text { for } & \mathbf{p}_{n} \neq \mathbf{p}_{k}^{*}
\end{array} .\right.
$$

From Eq.(5), $\mathbf{s}_{i} \in \mathbb{C}^{N}$ in the i sampling block can be also expressed as

$$
\mathbf{s}_{i}=\left[0, \cdots, s_{i 1}^{*}, 0, \cdots, s_{i k}^{*}, 0, \cdots, s_{i K}^{*}, 0, \cdots\right]_{N \times 1} .
$$

Since the number $\mathrm{K}$ of modeled source signals $\mathbf{s}^{*}$ is limited, and the number $\mathbf{N}$ of source signals $\mathbf{s}$ is much larger $(\mathrm{K}<\mathrm{N})$, so that $\mathbf{s}$ is a sparse signal with $\mathrm{K}$ sparsity in the space domain. Thus the estimation of $\mathbf{s}^{*}$ is transferred to estimate $\mathbf{s}$. After DFT transformation in time domain, $\mathbf{s}_{i}\left(f_{l}\right)$ still maintain the sparsity in space domain. The signal processing will be made independently for each frequency bin, thus in the following, we omit the frequency notation $f_{l}$ for simplicity.

In Eq.(1), replacing $\mathbf{P}^{*}$ by $\mathbf{P}$ and $\mathbf{s}_{i}^{*}$ by $\mathbf{s}_{i}$, we get the forward model of signal propagation based on the discretized source plane as follows:

$$
\mathbf{z}_{i}=\mathbf{A}(\mathbf{P}) \mathbf{s}_{i}+\mathbf{e}_{i}
$$


Remark that $i=1, \cdots, I$ denotes the index of sampling blocks; and $\mathbf{A}(\mathbf{P}) \in \mathbb{C}^{M \times N}$ consists of $\mathrm{N}$ steering vectors $\mathbf{a}\left(\mathbf{p}_{n}\right)$, which is similarly defined from Eq.(2) as follows:

$$
\mathbf{a}\left(\mathbf{p}_{n}\right)=\mathbf{a}_{d}\left(\mathbf{p}_{n}\right)+\rho \mathbf{a}_{r}\left(\mathbf{p}_{-n}\right)
$$

where $\mathbf{a}_{d}\left(\mathbf{p}_{n}\right)$ and $\mathbf{a}_{r}\left(\mathbf{p}_{-n}\right)$ are similarly defined from Eq.(3) and Eq.(4).

Therefore the forward model of Eq.(7) is the linear system of equations for source signals s. And we remark that source positions $\mathbf{P}^{*}$ can be estimated as the ones where the corresponding $\mathbf{s}$ are non-trivial values. However, since the source number $\mathrm{N}$ is usually larger than the sensor number $\mathrm{M}$, equation (7) thus is underdetermined, and some extra constraints should be involved in order to obtain stable and unique solutions of $\mathbf{s}$.

\subsection{Near-field beamforming}

Aeroacoustic imaging is actually focused on the source power estimation and location, rather than the source signal reconstruction. According to Eq.(7), the source powers can be obtained directly from the signals measured at sensors. Conventional beamforming (spatial filtering) is a signal processing technique used in phased sensor arrays for directional signal transmission or reception [7]. For the given location $\mathbf{p}_{n}$, the steering vector $\mathbf{a}\left(\mathbf{p}_{n}\right)$ of Eq.(8) is shortened to $\mathbf{a}_{n}$. Let $\tilde{y}_{n}$ denote the power estimation of the source signal $s_{n}$, thus $\tilde{y}_{n}$ can be expressed by the conventional beamforming method [4] as:

$$
\tilde{y}_{n}=\frac{\tilde{\mathbf{a}}_{n}^{H} \hat{\mathbf{R}} \tilde{\mathbf{a}}_{n}}{\left\|\tilde{\mathbf{a}}_{n}\right\|_{2}^{2}},
$$

where $\tilde{\mathbf{a}}_{n}=\frac{\mathbf{a}_{n}}{\left\|\mathbf{a}_{n}\right\|_{2}^{2}}$ denotes the near-field beamforming coefficient; and $\hat{\mathbf{R}}$ denotes the estimation of pressure cross variance matrix $\mathbf{R}$. In practice, $\hat{\mathbf{R}}$ is estimated by:

$$
\hat{\mathbf{R}}=\frac{1}{I} \sum_{i=1}^{I} \hat{\mathbf{z}}_{i} \hat{\mathbf{z}}_{i}^{H},
$$

where $\hat{\mathbf{z}}_{i}$ denotes the measured signals at the sensor array in the $i$ th sampling block, and I denotes the number of samplings blocks.

\subsection{Deconvolution method}

According to the discretized forward model of signal propagation in Eq.(7) and the independence assumptions in subsection 2.1, thus $\mathbf{R}$ is modeled by

$$
\mathbf{R}=\mathbb{E}\left\{\mathbf{z}_{i} \mathbf{z}_{i}^{H}\right\}=\mathbf{A} \mathbf{X} \mathbf{A}^{H}+\sigma^{2} \mathbf{I}_{M},
$$

where $\sigma^{2}$ denotes the variance of the i.i.d AGWN noise; $\mathbf{I}_{M}$ denotes the $\mathbf{M} \times \mathbf{M}$ identity matrix; and $\mathbf{X}$ denotes the cross spectrum matrix of the source signals, which is defined as $\mathbf{X}=\mathbb{E}\left\{\mathbf{s}_{i} \mathbf{s}_{i}^{H}\right\}$. The estimation of $\mathbf{X}$ has been directly solved by CMF method proposed in Ref.[17], in which, $\mathbf{X}$ can be reconstructed from Eq.(11), even if $\mathbf{X}$ is a nondiagonal matrix for the correlated sources. For uncorrelated source signals, $\mathbf{X}$ is a diagonal matrix. Let $\mathbf{x}$ denote the diagonal items of $\mathbf{X}$, defined as $\mathbf{x}=\operatorname{diag}[\mathbf{X}]$, thus $\mathbf{x}$ stands for the source powers, which is the final object for aeroacoustic imaging. For uncorrelated sources, we then get:

$$
\mathbf{R}=\sum_{q=1}^{N} x_{q} \mathbf{a}_{q} \mathbf{a}_{q}^{H}+\sigma^{2} \mathbf{I}_{M},
$$

where $\mathbf{a}_{q}$ is the steering vector of $\mathbf{A}$, defined in Eq.(8), and $x_{q} \in \mathbf{x}$ denotes the power at position $\mathbf{p}_{q}$, with $q=1, \cdots, N$.

Based on the near-field beamforming of Eq.(9), $\hat{\mathbf{R}}$ of Eq.(10) is known to converge to $\mathbf{R}$ of Eq.(11), as long as the sampling block number I is large enough, namely $\hat{\mathbf{R}} \approx \mathbf{R}$ for I $>>1$. Then introducing Eq.(12) into Eq.(9) and, match the following conditions: $\mathrm{I}>>1, \mathbf{X}$ is diagonal and particularly $\sigma^{2}=0$, we then obtain the Deconvolution Approach for Mapping of Acoustic Source (DAMAS) method (see [14] for details):

$$
\tilde{y}_{n}=\frac{\tilde{\mathbf{a}}_{n}^{H} \hat{\mathbf{R}} \tilde{\mathbf{a}}_{n}}{\left\|\tilde{\mathbf{a}}_{n}\right\|_{2}^{2}}=\sum_{q=1}^{N} c_{n, q} x_{q},
$$


where $c_{n, q}$ is known and it depends on the array geometry as follows:

$$
c_{n, q}=\frac{\left\|\tilde{\mathbf{a}}_{n}^{H} \mathbf{a}_{q}\right\|_{2}^{2}}{\left\|\tilde{\mathbf{a}}_{n}\right\|_{2}^{2}} .
$$

In Eq.(13), the beamforming estimated power $\tilde{y}_{n}$ for $x_{n}$ depends on all powers of vector $\mathbf{x}$, except when the microphone array aperture is large enough, the array response thus approximates the Dirac function. Namely, $x_{n}=\tilde{y}_{n}$ under condition of $c_{n, q} \approx \delta_{n, q}$ with $\delta_{n, q}=1$ for $n=q ; \delta_{n, q}=0$ for $n \neq q$. In practice, however, this condition is not verified. For vector $\mathbf{x}=\left[x_{1}, \cdots, x_{N}\right]^{T}$, Eq.(13) can be written in vectorial form as:

$$
\tilde{\mathbf{y}} \approx \mathbf{C x},
$$

where $\tilde{\mathbf{y}}=\left[\tilde{y}_{1}, \cdots, \tilde{y}_{N}\right]^{T}, \tilde{\mathbf{y}} \in \mathbb{R}^{N}$, which are derived by the beamforming in Eq.(9); and the power propagation matrix $\mathbf{C} \in \mathbb{R}^{N \times N}$ has the coefficient $c_{n, q}$ of Eq.(14). Since both $\mathbf{x}$ and $\tilde{\mathbf{y}}$ denote the powers, equation (15) could be regarded as the forward model of aeroacoustic power propagation.

Comparing to underdetermined Eq.(7) for source signals s, equation (15) becomes a determined linear system of equations for source power $\mathbf{x}$. But $N \times N$ matrix $\mathbf{C}$ is often singular as discussed in [14, 26], therefore DAMAS method has been proposed to iteratively solve Eq.(15) under non-negative constraint $(\mathbf{x} \geq 0)$. And it has been proved to obtain much higher spatial resolution than the beamforming method; however, DAMAS is not robust to the background noises due to assuming $\sigma^{2}=0$ in Eq.(12). The Diagonal Removal (DR) DAMAS [14] is thus proposed to set $\operatorname{diag}[R]=0$ for noise suppression, but DR technique inevitably harms weak source powers which are lower than the noise.

\section{Proposed forward model of aeroacoustic power propagation}

Recently, the Spectral Estimation Method (SEM) [24, 27] has been proposed to improve the robustness in aeroacoustic imaging,. SEM takes a known background noises as the reference, then subtracts the noise power from the measured data. And this reference noise could be estimated by measuring the observed signals without any object in wind tunnel. However, the estimated noise power might not be the same one when the object exists in the wind tunnel.

\subsection{Improved forward model}

In this paper, we intend to simultaneously estimate the source powers, background noise powers. Moreover, we consider the forward model uncertainty which can be caused by the acoustic multipath propagations in wind tunnel experiments, such as the reverberations, the reflections on the ground, as well as the refractions on the interface between wind and common air, etc. Even though both reflections and refractions could be reduced to some extent, as discussed in Appendix C and Appendix D, forward model uncertainty could hardly be exactly modeled and completely removed off. Therefore it is necessary to consider the forward model uncertainty to be a random variable vector. Compared to forward model uncertainty, background noises naturally exist at the sensors due to antenna properties, and are supposed as i.i.d AGWN in Assumptions of Section 2.1. And we also assume the mutual independence among the sources, background noises and forward model uncertainty. For the uncorrelated sources, we introduce Eq.(12) into Eq.(9), then we obtain the improved forward model of aeroacoustic power propagation as follows:

$$
\mathbf{y}=\mathbf{C x}+\xi+\sigma^{2} 1_{N}
$$

where $\mathbf{y}$ denotes the modeled output powers at sensor array; $\sigma^{2}$ denotes the variance of the i.i.d. AGWN noises at the sensor array; and $\xi=\left[\xi_{1}, \cdots, \xi_{N}\right]^{T}$ denotes the forward model uncertainty caused by multipath propagations; and $1_{N}=[1, \cdots, 1]_{N}^{T}$.

\subsection{Classical regularization methods}

For the inverse solution of Eq.(16), a classical approach can introduce the regularization part to improve the resolution and robustness, then the criterion can be to minimize both the regularization part and forward model uncertainty $\boldsymbol{\xi}$ :

$$
\left\{\begin{array}{l}
\left(\hat{\mathbf{x}}, \hat{\sigma}^{2}\right)=\arg \min _{\left(\mathbf{x}, \sigma^{2}\right)}\left\{\left\|\mathbf{y}-\mathbf{C x}-\sigma^{2} 1_{N}\right\|_{2}^{2}+\alpha \mathcal{F}(\mathbf{x})\right\} \\
\text { s.t. } \quad \mathbf{x} \geq 0, \sigma^{2} \geq 0
\end{array} .\right.
$$


where the first term $\|\cdot\|^{2}$ represents the data fitting part, which aims to minimize the power of forward model uncertainty. In practice, the modeled output powers $\mathbf{y}$ are known as the measured beamforming powers $\tilde{\mathbf{y}}$ from Eq.(9).

The second term $\mathcal{F}(\cdot)$ usually takes the following forms: 1) $\mathcal{F}(\mathbf{x})=\|\mathbf{x}\|_{0}$ refers to the number of non-zero elements of $\mathbf{x}$, which can give the sparsest solution; unfortunately, $\|\mathbf{x}\|_{0}$ is impossible to find stable solutions for large dimension $\mathbf{x}$, even if the Iterative Hard Thresholding (IHT) method have been proposed recently [28]; 2) $\mathcal{F}(\mathbf{x})=\|\mathbf{x}\|_{1}$ involves the $\ell_{1}$ regularization, which can enforce sparse solution; and $\ell_{1}$ regularization can be solved by the LASSO in [29] and atomic decomposition by basis pursuit in [30]; 3) $\mathcal{F}(\mathbf{x})=\|\mathbf{x}\|_{l}$ with $0<l<1$ corresponds to the Iterative Reweighed algorithm [31], which obtains sparser reconstruction of source powers than the $\ell_{1}$ regularization does, but $0<l<1$ involves the non-convex optimization problem; 4) $\mathcal{F}(\mathbf{x})=\|\mathcal{D} \mathbf{x}\|_{2}^{2}$ refers to the Tikhonov regularization [32], where the operator $\mathcal{D}$ can suppress the noise interference, but $\|\cdot\|_{2}^{2}$ can not offer as high resolution as the $\ell_{1}$ regularization does.

The third term $\alpha$ denotes regularization parameter. Tuning $\alpha$ is an important issue, and various empirical approaches $[30,32,33]$ have been proposed. For example, optimal Tikhonov regularization parameter is selected via generalized cross validation and L-curve method in paper [32]. However, we expect that $\alpha$ should be automatically determined through the estimations in order to insure the global optimization.

\section{Proposed robust Bayesian super-resolution approach via sparsity enforcing a priori}

In order to adaptively find a sparse and robust solution for improved forward model of power propagation in Eq.(16), we propose a robust Bayesian super-resolution approach via sparsity enforcing a priori by jointly estimating the source powers and positions, as well as background noises and hyperparameters of prior models.

Bayesian inference $[34,35]$ is a powerful methodology for solving ill-posed inverse problem. It aims to estimate the unknown variables by applying the Bayes' rule to update the probability law: an a posteriori probability law is obtained based on both the likelihood and a priori, in which, the previous one can be derived from the known data and forward model, and the latter one can be imposed to unknown variables. Comparing to determined methods, the priors in statistics can bring in novel information on the natural characteristics of unknown variables, which can also reduce the uncertainty of ill-posed inverse problem.

Starting with our improved forward model of Eq.(16), we can assign the likelihood $p(\mathbf{y} \mid \mathbf{x})$ based on the basic information of forward model uncertainty $\boldsymbol{\xi}$. Generally, $\boldsymbol{\xi}$ is supposed to be Gaussian distribution as $\boldsymbol{\xi} \sim \mathcal{N}\left(0, \sigma_{\xi}^{2}\right)$, where $\sigma_{\xi}^{2}$ represents the power of forward model uncertainty. Thus the likelihood $p\left(\mathbf{y} \mid \mathbf{x}, \boldsymbol{\theta}_{1}\right)$ is obtained as:

$$
p\left(\mathbf{y} \mid \mathbf{x}, \boldsymbol{\theta}_{1}\right)=\frac{1}{\left(2 \pi \sigma_{\xi}^{2}\right)^{N / 2}} \exp \left\{-\frac{\left\|\mathbf{y}-\mathbf{C x}-\sigma^{2} 1_{N}\right\|^{2}}{2 \sigma_{\xi}^{2}}\right\} .
$$

where unknown parameters $\boldsymbol{\theta}_{1}=\left[\sigma^{2}, \sigma_{\xi}^{2}\right]^{T}$ denotes the hyperparameters of the above likelihood, and $\boldsymbol{\theta}_{1}$ are independent to source powers $\mathbf{x}$; and $p(\cdot)$ denotes the probability density function (PDF). In practice, $\mathbf{y}$ are known as the measured beamforming powers $\tilde{\mathbf{y}}$ from Eq.(9).

Let $\boldsymbol{\theta}=\left[\boldsymbol{\theta}_{1}, \boldsymbol{\theta}_{2}\right]^{T}$ denote the hyperparameters to be estimated, with $\boldsymbol{\theta}_{1}$ being likelihood hyperparameters and $\boldsymbol{\theta}_{2}$ being the prior hyperparameters. And assume $\boldsymbol{\theta}=\left[\boldsymbol{\theta}_{1}, \boldsymbol{\theta}_{2}\right]^{T}$ and source powers $\mathbf{x}$ are mutually independent to each other. Now if we can assign a prior law $p\left(\mathbf{x} \mid \boldsymbol{\theta}_{2}\right)$ on $\mathbf{x}$, we can obtain $p(\mathbf{x} \mid \mathbf{y}, \boldsymbol{\theta}) \propto p\left(\mathbf{y} \mid \mathbf{x}, \boldsymbol{\theta}_{1}\right) p\left(\mathbf{x} \mid \boldsymbol{\theta}_{2}\right)$ using the Bayes' rule $p(\mathbf{x} \mid \mathbf{y}, \boldsymbol{\theta})=p\left(\mathbf{y} \mid \mathbf{x}, \boldsymbol{\theta}_{1}\right) p\left(\mathbf{x} \mid \boldsymbol{\theta}_{2}\right) / p(\mathbf{y})$ with $p(\mathbf{y})$ being a constant for the observed data $\mathbf{y}$. Then if we apply the Maximum a Posteriori (MAP) criterion, we can obtain $\hat{\mathbf{x}}=\arg \max _{(\mathbf{x})}\{p(\mathbf{x} \mid \mathbf{y}, \boldsymbol{\theta})\}=\arg \min _{(\mathbf{x})}\left\{-\ln p\left(\mathbf{y} \mid \mathbf{x}, \boldsymbol{\theta}_{1}\right)-\ln p\left(\mathbf{x} \mid \boldsymbol{\theta}_{2}\right)\right\}$, where In denotes Logarithm operator. Then if $\boldsymbol{\theta}$ is unknown, we can also consider it as an extra unknown variable and try to estimate it by defining the joint posterior: $p(\mathbf{x}, \boldsymbol{\theta} \mid \mathbf{y}) \propto p\left(\mathbf{y} \mid \mathbf{x}, \boldsymbol{\theta}_{1}\right) p(\mathbf{x}, \boldsymbol{\theta})$; since $\mathbf{x}$ and $\boldsymbol{\theta}$ are mutually independent to each other as $p(\mathbf{x}, \boldsymbol{\theta})=p\left(\mathbf{x} \mid \boldsymbol{\theta}_{2}\right) p\left(\boldsymbol{\theta}_{2}\right) p\left(\boldsymbol{\theta}_{1}\right)$, the proposed joint MAP criterion is thus given as follows:

$$
\left\{\begin{array}{l}
p(\mathbf{x}, \boldsymbol{\theta} \mid \mathbf{y}) \propto p\left(\mathbf{y} \mid \mathbf{x}, \boldsymbol{\theta}_{1}\right) p(\mathbf{x}, \boldsymbol{\theta})=p\left(\mathbf{y} \mid \mathbf{x}, \boldsymbol{\theta}_{1}\right) p\left(\mathbf{x} \mid \boldsymbol{\theta}_{2}\right) p\left(\boldsymbol{\theta}_{2}\right) p\left(\boldsymbol{\theta}_{1}\right) \\
(\hat{\mathbf{x}}, \hat{\boldsymbol{\theta}})=\arg \max _{(\mathbf{x}, \boldsymbol{\theta})}\{p(\mathbf{x}, \boldsymbol{\theta} \mid \mathbf{y})\}=\arg \min _{(\mathbf{x}, \boldsymbol{\theta})}\left\{-\ln p\left(\mathbf{y} \mid \mathbf{x}, \boldsymbol{\theta}_{1}\right)-\ln p\left(\mathbf{x} \mid \boldsymbol{\theta}_{2}\right)-\ln p\left(\boldsymbol{\theta}_{2}\right)-\ln p\left(\boldsymbol{\theta}_{1}\right)\right\}
\end{array},\right.
$$

where $-\ln p\left(\mathbf{x} \mid \boldsymbol{\theta}_{2}\right)$ can be interpreted as the regularization form $\mathcal{F}(\cdot)$ in Eq.(17), and hyperparameter $\boldsymbol{\theta}_{2}$ takes the similar effect as the regularization parameter does in Eq.(17). Indeed, one of the advantages of Bayesian inference approach is that hyperparameters $\boldsymbol{\theta}=\left[\boldsymbol{\theta}_{1}, \boldsymbol{\theta}_{2}\right]^{T}$ can be jointly estimated by joint MAP in Eq.(19). 
For the prior $p\left(\mathbf{x} \mid \boldsymbol{\theta}_{2}\right)$ on source powers $\mathbf{x}$, we reconsider the sparsity fact that aeroacoustic sources sparsely lay on the surface of the object, and the source number $\mathrm{K}$ is relatively much smaller with respect to the total scanning points $\mathrm{N}$ on the source plane. Taking the vehicle in the wind tunnel test as shown in Fig.1a and 2a for example, most of sources mainly locate on the particular parts the rearview mirrors and wheels, whereas for the rest parts, there are few sources existing. And such a sparse distribution can be represented by a centralized PDF function that has a very high value around the original zero (sparsity) and a long heavy tail (dynamic range of source powers). This kind of centralized PDF function can be selected among the Generalized Gaussian family, see Appendix A for details. At last, we take the Double Exponential $\mathcal{D E}(\mathbf{x})$ model as the sparse a priori as follows:

$$
p\left(\mathbf{x} \mid \boldsymbol{\theta}_{2}\right)=\prod_{n=1}^{N} \mathcal{D} \mathcal{E}\left(x_{n} \mid \gamma\right)=\left(\frac{\gamma}{2}\right)^{N} \exp \left\{-\gamma\|\mathbf{x}\|_{\beta=1}\right\},
$$

where $\boldsymbol{\theta}_{2}=\gamma$ is the scale parameter, which controls the dynamic range of $\mathbf{x}$, and $\|\mathbf{x}\|_{\beta=1}$ represents the $l_{1}$ norm, which can promote the sparsity of $\mathbf{x}$.

For hyperparameters $\boldsymbol{\theta}=\left[\boldsymbol{\theta}_{1}, \boldsymbol{\theta}_{2}\right]^{T}$ in Eq.(19), we have $\boldsymbol{\theta}_{1}=\left[\sigma^{2}, \sigma_{\xi}^{2}\right]^{T}, \boldsymbol{\theta}_{2}=\gamma$. Assuming the independence among hyperparameters, we have $p(\boldsymbol{\theta})=p\left(\sigma^{2}\right) p\left(\sigma_{\xi}^{2}\right) p(\gamma)$. Since these parameters are non-negative, we take Jeffreys priors [36] for simplicity as follows:

$$
p\left(\sigma^{2}\right) \sim \frac{1}{\sigma^{2}}, \quad p\left(\sigma_{\xi}^{2}\right) \sim \frac{1}{\sigma_{\xi}^{2}}, \quad p(\gamma) \sim \frac{1}{\gamma} .
$$

Here we mark one of the advantages of Jeffreys priors, take $p(\gamma) \sim \frac{1}{\gamma}$ for instance, Jeffreys prior model does not bring in the extra hyperparameters which have to be selected carefully to describe the scale parameter $\gamma$.

\subsection{Hyperparameter estimations by using Bayesian inference approach}

There are several classical methods [37-40] to solve the joint posterior probability in Eq.(19). Here we choose the joint MAP [40] optimization, since joint MAP can build up a relationship with the classical regularization method in Eq.(17). Based on the selections of prior models in the above, we take the followings into the Eq.(19): the likelihood $p\left(\mathbf{y} \mid \mathbf{x}, \boldsymbol{\theta}_{1}\right)$ in Eq.(18), the sparsity enforcing a priori $p\left(\mathbf{x} \mid \boldsymbol{\theta}_{2}\right)$ in Eq.(20), and Jeffreys priors $p(\boldsymbol{\theta})$ of parameters in Eq.(21). By omitting trivial value terms and considering the non-negative constraints of source powers and hyperparameters, our proposed Bayesian approach is obtained as follows:

$$
\left\{\begin{array}{l}
(\hat{\mathbf{x}}, \hat{\boldsymbol{\theta}})=\arg \min _{(\mathbf{x}, \boldsymbol{\theta})}\{\mathcal{J}(\mathbf{x}, \boldsymbol{\theta})\} \\
\mathcal{J}(\mathbf{x}, \boldsymbol{\theta})=\frac{1}{2 \sigma_{\xi}^{2}}\left\|\mathbf{y}-\mathbf{C x}-\sigma^{2} 1_{N}\right\|^{2}+\gamma\|\mathbf{x}\|_{1}+\frac{N}{2} \ln \sigma_{\xi}^{2}-N \ln \gamma \\
\text { s.t. } \quad \mathbf{x} \geq 0, \sigma^{2} \geq 0, \sigma_{\xi}^{2} \geq 0, \gamma \geq 0
\end{array} .\right.
$$

where hyperparameters $\boldsymbol{\theta}=\left[\sigma^{2}, \sigma_{\xi}^{2}, \gamma\right]^{T} ; \mathbf{y}$ are known as the measured beamforming powers $\tilde{\mathbf{y}}$ from Eq.(9); In the cost function $\mathcal{J}(\mathbf{x}, \boldsymbol{\theta})$, the first term represents the data fitting; the second term represents the $\ell_{1}$ sparse regularization, and $\gamma$ performs as the regularization parameter; the rest terms are derived from the priors $p(\boldsymbol{\xi})$, as well as the sparse prior $p(\mathbf{x} \mid \gamma)$; particularly, the joint MAP optimization in Eq.(22) reveals that $\gamma$ should not be neither too big nor too small, and its proper value can not only enforce the sparsity of source power distributions, but also can promote the dynamic range of estimated source powers. For hyperparameter estimations in Eq.(22), we use an alternative optimization procedure as discussed in Appendix B: we firstly initialize $\mathbf{x}$, then at any iteration $\mathrm{k}+1$, we obtain a new value for $\boldsymbol{\theta}$ by minimizing $\mathcal{J}\left(\hat{\mathbf{x}}^{(k)}, \boldsymbol{\theta}\right)$ with respect to $\boldsymbol{\theta}$; At the next iteration we alternatively update $\mathbf{x}$ by minimizing $\mathcal{J}\left(\mathbf{x}, \hat{\boldsymbol{\theta}}^{(k)}\right)$ with respect to $\mathbf{x}$; Finally we repeat this alternative optimization procedure until $\mathcal{J}(\mathbf{x}, \boldsymbol{\theta})$ converges.

We have to point out 4 advantages with respect to classical methods: 1) estimation of $\sigma_{\xi}^{2}$ and $\sigma^{2}$ improves the robustness to the forward model uncertainty and noise interference; 2) applying $\ell_{1}$ norm of $\|\mathbf{x}\|_{1}$ enforces sparsity of $\mathbf{x}$, and achieve a super spatial resolution of power distributions; 3) estimation of $\gamma$ provides a wide dynamic range of source powers, moreover, $\gamma$ takes the similar effect as the regularization parameter $\alpha$ does in Eq.(17); 4) $\mathbf{x}$ and hyperparameters are alternatively estimated without knowing the source number or SNR. 


\subsection{Relation with regularization approach}

Furthermore, comparing to the classical methods of Eq.(17), the regularization parameter can be expressed in function of hyperparameters in the proposed Bayesian approach of Eq.(22) as follows:

$$
\alpha=2 \sigma_{\xi}^{2} \gamma,
$$

where we can see that $\alpha$ is proportional to $\sigma_{\xi}^{2}$ and $\gamma$. Particularly when Gaussian prior in Eq.(A.3) in Appendix A is used $\left(\gamma=\frac{1}{2 \sigma_{x}^{2}}\right)$ on source powers, $\alpha=\frac{\sigma_{\xi}^{2}}{\sigma_{x}^{2}}$ means the inverse of signal-to-uncertainty ratio. Since we can take the forward model uncertainty as the background noises to some extent, $\alpha$ equals the inverse of SNR. Our conclusion agrees with the argument in paper [30]. Comparing to the classical regularization methods in Eq.(17), our proposed Bayesian approach has the advantage of automatically estimating the regularization parameter.

\subsection{Estimation of wide-band aeroacoustic powers}

In wind tunnel experiments, aeroacoustic sources are generated by the friction between the car and wind flow. Physically, different car parts produce the vibrations with different frequencies. Therefore aeroacoustic sources are of wide-band signals. We now consider the frequency range $\left[f_{\min }, f_{\max }\right]$ consisting of $L$ frequency bins. Let $\hat{\mathbf{x}}\left(f_{l}\right)$ be the estimation of $\mathbf{x}\left(f_{l}\right)$ in $l$ th frequency bin. Then total power $\mathbf{x}_{w b}$ over the band $\left[f_{\min }, f_{\max }\right]$ can be estimated by $\hat{\mathbf{x}}_{w b}=\sum_{f_{l}=f_{\min }}^{f_{\max }} \hat{\mathbf{x}}\left(f_{l}\right)$, where $\hat{\mathbf{x}}\left(f_{l}\right)$ denotes the estimated power at frequency $f_{l}$, and $\hat{\mathbf{x}}\left(f_{l}\right)$ can be estimated from our proposed Bayesian approach in Eq.(22). We remark that in the beginning of the section 2.2, we have taken DFT transformation in time domain and made analysis at the single frequency.

\section{Simulated data of monopole and extended sources}

This section demonstrates the performances of the proposed Bayesian approach for near-field aeroacoustic imaging in the poor SNR cases. The proposed Bayesian approach is compared with 6 classical methods mentioned in the above. Reconstruction results are presented on images which will directly demonstrate the estimated source positions, and the estimated source powers $(\mathrm{dB})$. The dynamic range of estimated powers is presented by the colormap with $15 \mathrm{~dB}$ span. In addition, section profiles are shown on the edge of the images, which are used to show the estimated positions of particular sources. Then we use two criteria $\overline{\Delta x^{*}}$ and $\delta_{i}$ to quantitatively evaluate the precision of source powers and positions estimation. One is the averaged estimation error of modeled source power, defined by $\overline{\Delta x^{*}}=\frac{1}{K} \sum_{k=1}^{K}\left|\hat{x}_{k}^{*}-x_{k}^{*}\right|$. And we remark that modeled source powers $\mathbf{x}^{*}=\left[x_{1}^{*}, \cdots, x_{K}^{*}\right]^{T}$ are obtained by $\mathbf{x}^{*}=\operatorname{diag}\left[\mathbb{E}\left\{\mathbf{s}^{*} \mathbf{s}^{* H}\right\}\right]$. The other is the relative error of power image reconstruction, defined by $\delta_{i}=\frac{\|\hat{\mathbf{x}}-\mathbf{x}\|_{i}^{i}}{\|\mathbf{x}\|_{i}^{i}}$, with $i=1,2$. Compared to $\overline{\Delta x^{*}}$, the value of $\delta_{i}$ not only depends on the estimated positions and powers, but also relies on the estimation of background noises.

\subsection{Simulation configurations}

In order to well fit for the conditions of wind tunnel S2A in Fig.2, the configurations of simulations are the same as the those in the wind tunnel experiments carried out by Renault SAS [41]. For example, $D=4.50 \mathrm{~m}$ is the distance between the sensor plane and source plane. There are M= 64 non-uniform sensors locating on the $2 \times 4 \mathrm{~m}^{2}$ vertical plane. The NUA array has the longer horizontal aperture than the vertical one, as shown in Fig.2a. The advantage of NUA array is that with fewer sensors, NUA array yields almost the same performance as the uniform array with larger number of sensors, and this advantage can lower computation burden as discussed in [42]. And $d=2 \mathrm{~m}$ is the average aperture of sensor array. $c_{0} \approx 340 \mathrm{~m} / \mathrm{s}$ is the aeroacoustic propagation speed in the open air. $T=10000$ is the total number of samplings, which is large enough to fit for the important condition in Eq.(13).

For the simulated sources in Fig.3a, we have simulated 4 monopole sources and 5 extended sources with different patterns, and the source number of total monopoles is $\mathrm{K}=23$; their powers $\mathbf{x}$ are within $[0.08,2]([-10.3,3.7] \mathrm{dB})$ with $14 \mathrm{~dB}$ dynamic range. We mark that there are 4 parts on Fig.3a: the center image shows the source positions, patterns and powers. On its right, the colormap shows the power level by using different colors, in which, the darker red colors represent stronger powers, and lighter white colors represent weak powers. On its left and bottom, 2 profile figures show the powers of the 4 monopole sources and one of the extended sources. To simulate the very noisy background, we take $\sigma^{2}=0.86(-0.7 \mathrm{~dB})$ as the noise power, then the average SNR is $0 \mathrm{~dB}$. 


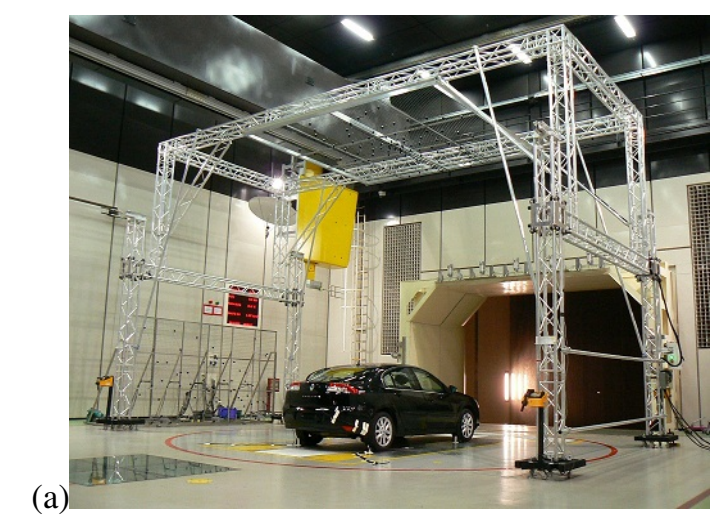

(a) (b)

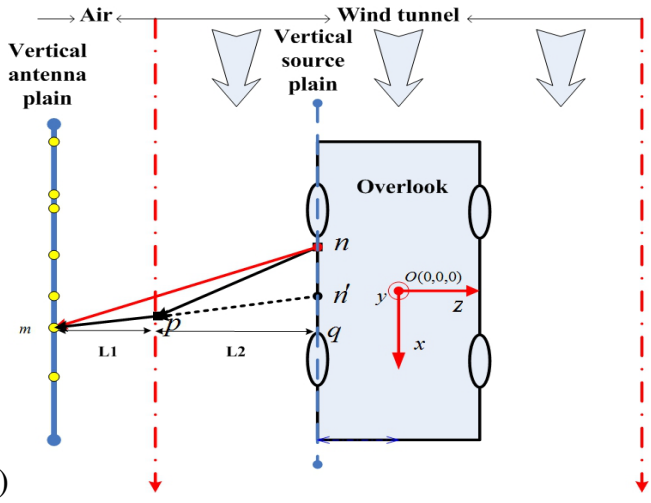

Figure 2: Configurations of wind tunnel S2A: (a) wind tunnel S2A [41] and (b) overlook and wind refraction.

In order to well compare with other classical methods, some simulation parameters should be selected carefully. Near-field condition is guaranteed by $D<d^{2} /(4 \lambda)$ for any $f>1500 \mathrm{~Hz}$. Since the spatial resolution of beamforming at $\mathrm{f}=2500 \mathrm{~Hz}$ is $\Delta B \approx \lambda R / d=31 \mathrm{~cm}$, the selected scanning step $\Delta p=5 \mathrm{~cm}$ satisfies $\Delta p / \Delta B<0.2$ for any $f<3500 \mathrm{~Hz}$, which can avoid the spatial aliasing problem as discussed in the DAMAS [14]. For the region of $1 \times 1.5 \mathrm{~m}^{2}$, the scanning image is of $21 \times 31$ pixels. Since the scanning point number $N=651$ is much more larger than source number $K=23$, the simulated source signals $\mathbf{s}$ and source powers $\mathbf{x}$ in Fig.3a are both K-sparsity signals.

\subsection{Method comparisons}

In this part, we firstly show the reconstruction results of source power images. Then we make quantitative comparisons of different methods. Finally we further validate our proposed Bayesian approach by simulations in the cases of various noise levels and different frequency bins.

Figure 3 shows the reconstruction results of simulated data at $\mathrm{f}=2500 \mathrm{~Hz}$ and $\mathrm{SNR}=0 \mathrm{~dB}$. In Fig. $3 \mathrm{~b}$, the beamforming [4] method merely gives a very fussy result of strong sources due to its low spatial resolution $(\Delta B \approx 31 \mathrm{~cm}$ at $2500 \mathrm{~Hz}$ ). In Fig.3c and d, both the DAMAS [14] with 5000 iterations (5000i) and CLEAN [13] could not provide reliable reconstructions in strong background noises. This can be explained by the fact that DAMAS omits the background noises as discussed in Eq.(15); As for CLEAN, it iteratively subtracts the maximal source power from the original beamforming result in Fig.3b, but CLEAN might not easily detect the weak sources whose powers are lower than the noise, moreover, some important parameters such as the attenuation factor and iteration number have to be selected carefully by CLEAN. In Fig.3e, DR-DAMAS [14] removes the noise influence, and roughly estimates the extended source, but it loses some of weak sources, this is because the diagonal removal operation distracts both the noise and weak sources. The CMF [17] aims to reconstruct both the spectrum covariance matrix $\mathbf{R}$ and the noise power in Eq.(11) under the sparsity constraint. In Fig.3f, CMF well estimates the noise power and distinguishes most of the sources, however, it also fails to find weak sources, the reason might be that the adaptiveness of CMF method depends on an important parameter of the sparsity constraint. Comparing to CMF, the SC-RDAMAS [21] in Fig.3g achieves better reconstructions, since SC-RDAMAS directly estimates the source powers and background noises, and it also adaptively estimates the sparsity parameter according to the noise level. But the performance of SC-RDAMAS still relies on the source number estimation. In order to make a fair comparison, both CMF and SC-RDAMAS are realized by the MATLAB toolbox SeMuDi [43].

In Fig.3h, the proposed Bayesian inference approach works much better than the above methods regardless of source patterns and positions. For the monopole sources, proposed approach not only accurately estimate their positions, but also detects the weak sources as well as strong sources; for the extended sources, it successfully reconstructs both source powers and different patterns. The dynamic range of estimated powers is achieved as large as $14 \mathrm{~dB}$. Comparing to the beamforming resolution $(31 \mathrm{~cm})$ [4], proposed approach achieves the $5 \mathrm{~cm}$ super-resolution, thanks to the contribution of applying $\mathcal{D} \mathcal{E}(\mathbf{x})$ sparse prior in Eq.(20). In addition, proposed approach is more robust to noise interference than the deconvolution methods (DAMAS, DR-DAMAS [14] and CLEAN [12]). This is owing to the simultaneous estimation of the background noises $\sigma^{2}$ and forward model uncertainty $\sigma_{\xi}^{2}$, as well as source powers $\mathbf{x}$. 


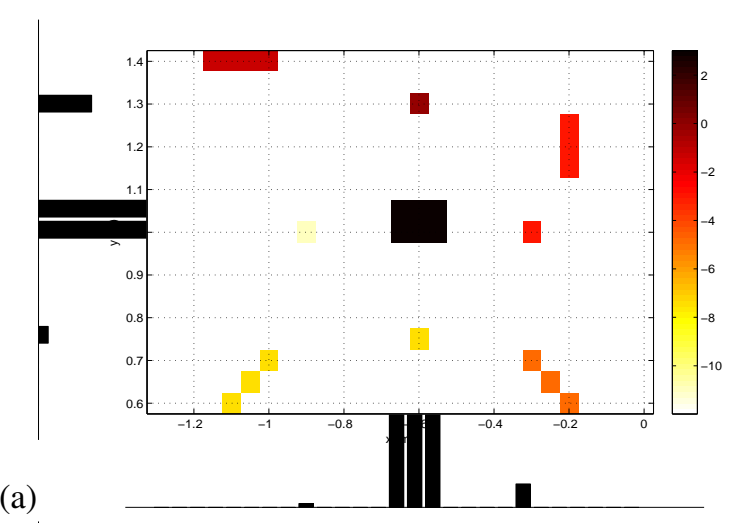

(a)
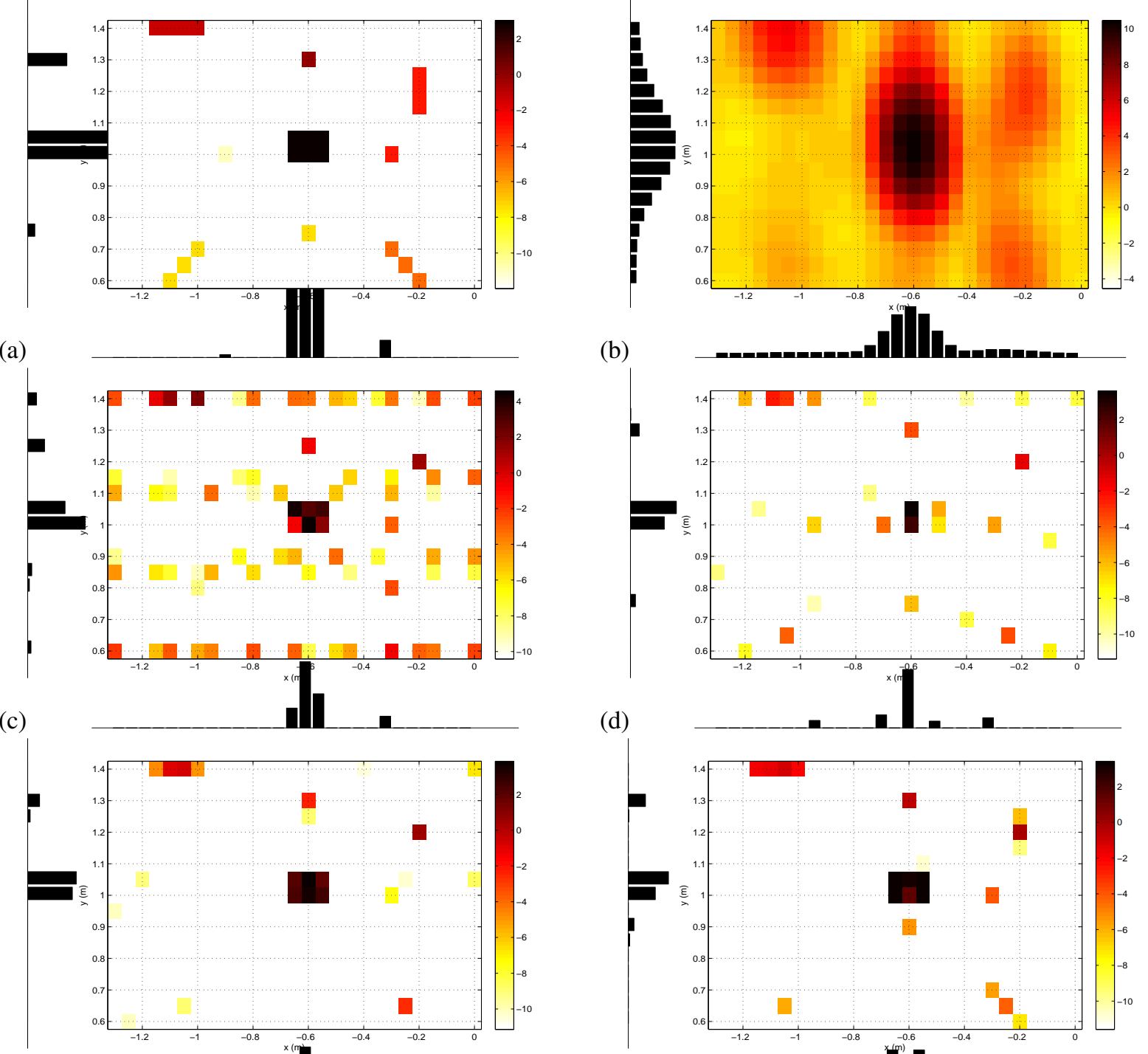

(d)

(e)

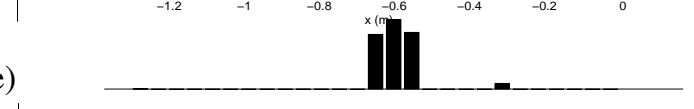

(f)
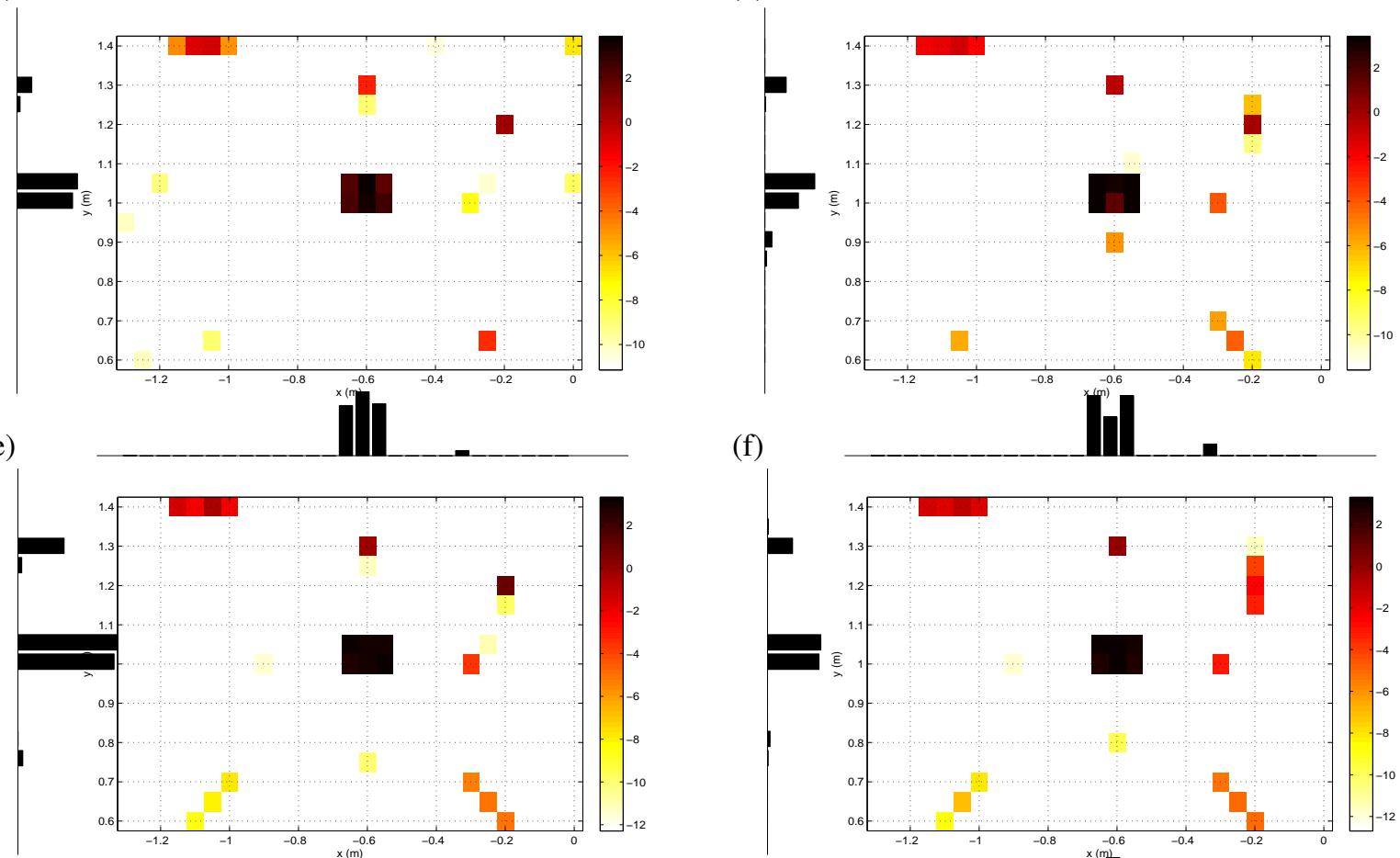

(g)

(h)
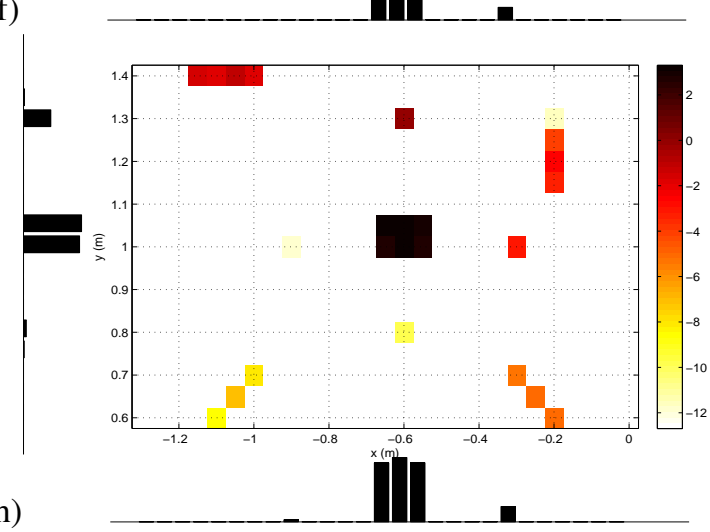

Figure 3: Simulation on extended sources with $14 \mathrm{~dB}$ power dynamic range at $2500 \mathrm{~Hz}$, real $\sigma^{2}=0.86$, $\mathrm{SNR}=0 \mathrm{~dB}$ and $15 \mathrm{~dB}$ display : (a) 5 extended sources and 4 monopoles (b) Beamforming (c) DAMAS with 5000 iterations (5000i) (d) CLEAN. (e) DR-DAMAS (5000i) (f) CMF (g) SC-RDAMAS and (h) Proposed Bayesian MAP approach 
These two important parameters are estimated as the hyperparameters in the joint MAP criterion of Eq.(22). Comparing to the adaptiveness of sparse regularization methods (CMF [17] and SC-RDAMAS [21]), the scale parameter $\gamma$ in proposed approach has the similar effect as the sparsity parameter. Since proposed Bayesian inference approach adaptively estimates $\gamma$ from the the hyperparameter estimations, we provide a high resolution reconstruction with large dynamic range of estimated powers, even in the low SNR case.

The hyperparameter $\left(\theta=\left[\sigma^{2}, \sigma_{\xi}^{2}, \gamma\right]^{T}\right)$ estimations offer the following results: the estimated variance of background noises is $\hat{\sigma}^{2}=0.86$, almost the same as the simulated $\sigma^{2}=0.86$; and $\hat{\sigma}_{\xi}^{2}=0.69$ is the estimated power of forward model uncertainty; this estimated result means that the propagation uncertainty is not very obvious, but indeed, $\hat{\sigma}_{\xi}^{2}=0.69$ can not be negligible with respect to the noise $\sigma^{2}=0.86$; the scale parameter $\hat{\gamma}$ in the sparse prior is the estimated as $\hat{\gamma}=0.72$; As illustrated in Fig.A.1 of Appendix A, parameter $0<\hat{\gamma}<1$ makes $\mathcal{D E}(\mathbf{x})$ model to have a much sparser distribution with a longer and heavier tail among the generated Gaussian distribution families, so that our proposed Bayesian inference approach manages to obtain both the sparse reconstructions of source powers $\mathbf{x}$ and the wide dynamic ranges of the estimated $\mathbf{x}$ at the same time.

To quantitatively validate our proposed Bayesian approach, table 1 and table 2 show the power estimations for monopole and extended sources, as well as the average estimation error $\overline{\Delta x *}$, and the relative error of power image reconstruction $\delta_{i}$. According to $\overline{\Delta x^{*}}$ and $\delta_{i}$, it is seen that our proposed method not only successfully detects each pattern of extended sources, but also better estimates source powers and background noises in very poor SNR situations.

Table 1: Power estimations of 4 monopole sources by average power estimation error $\overline{\Delta x^{*}}$, relative errors of power image reconstruction $\delta_{1}, \delta_{2}$ and estimated noise covariance $\hat{\sigma^{2}}$ at $2500 \mathrm{~Hz}, \mathrm{SNR}=0 \mathrm{~dB}$, dynamic range $14 \mathrm{~dB}$, a cell containing '-' means unavailable.

\begin{tabular}{|l|c|c|c|c|c|c|c|c|}
\hline Source powers & 0.08 & 0.18 & 0.98 & 0.50 & $\overline{\Delta x^{*}}$ & $\delta_{1}$ & $\delta_{2}$ & $\hat{\sigma}^{2}\left(\sigma^{2}=0.86\right)$ \\
\hline Beamforming & 1.57 & 11.28 & 3.51 & 2.02 & 4.16 & 69.64 & 121.93 & - \\
\hline DAMAS & - & - & - & 0.44 & 0.33 & 3.14 & 1.33 & - \\
\hline CLEAN & - & 0.25 & 0.44 & 0.28 & 0.23 & 0.87 & 0.67 & - \\
\hline DR-DAMAS & - & - & 0.77 & 0.23 & 0.19 & 0.30 & 0.08 & - \\
\hline CMF & 0.09 & - & 0.80 & 0.40 & 0.12 & 0.31 & 0.10 & 0.89 \\
\hline SC-RDAMAS & 0.09 & 0.10 & 1.05 & 0.43 & 0.06 & 0.21 & 0.06 & 0.85 \\
\hline Proposed & 0.08 & 0.13 & 0.94 & 0.45 & 0.04 & 0.17 & 0.02 & 0.86 \\
\hline
\end{tabular}

Table 2: Power estimations of the extended source on the center of image by power estimation error $\overline{\Delta x^{*}}$ at $2500 \mathrm{~Hz}, \mathrm{SNR}=0 \mathrm{~dB}$, a cell containing '-' means unavailable.

\begin{tabular}{|l|c|c|c|c|c|c|c|}
\hline Source powers & 2.00 & 2.00 & 2.00 & 2.00 & 2.00 & 2.00 & $\overline{\Delta x^{*}}$ \\
\hline Beamforming & 2.64 & 9.60 & 9.70 & 9.64 & 11.34 & 9.77 & 6.78 \\
\hline DAMAS & 4.50 & 1.25 & 0.48 & 2.54 & 0.49 & 1.88 & 1.15 \\
\hline CLEAN & 2.29 & 0.37 & 1.69 & - & 0.27 & 0.34 & 1.27 \\
\hline DR-DAMAS & 2.15 & 2.05 & 1.82 & 1.83 & 2.50 & 1.45 & 0.27 \\
\hline CMF & 1.36 & 2.86 & 2.07 & 2.09 & 1.92 & 1.05 & 0.45 \\
\hline SC-RDAMAS & 1.83 & 2.00 & 2.05 & 1.72 & 2.16 & 1.95 & 0.12 \\
\hline Proposed & 1.94 & 1.99 & 1.98 & 1.76 & 2.10 & 1.91 & 0.09 \\
\hline
\end{tabular}

Figure 4 and 5 show the simulations in the cases of various noise levels and different frequency bins. Each point on the curves is the averaged result based on 15 simulations. Firstly we fix the $\mathrm{f}=2500 \mathrm{~Hz}$. In Fig.4, the power image reconstruction errors $\delta_{i}$ with $i=1,2$ are plotted versus the $\mathrm{SNR} \in[-6,18] \mathrm{dB}$, and it shows the robustness to background noises of the mentioned methods. As we can see, the proposed Bayesian approach is very robust to background noises, and outperforms the other methods in the cases from -6dB to $18 \mathrm{~dB}$. Particularly in very poor SNR cases, it still achieves very small $\delta_{1}$ and $\delta_{2}$. Then we fix the $\mathrm{SNR}=3 \mathrm{~dB}$. In Fig.5, $\delta_{i}$ are plotted versus frequencies 
(a)

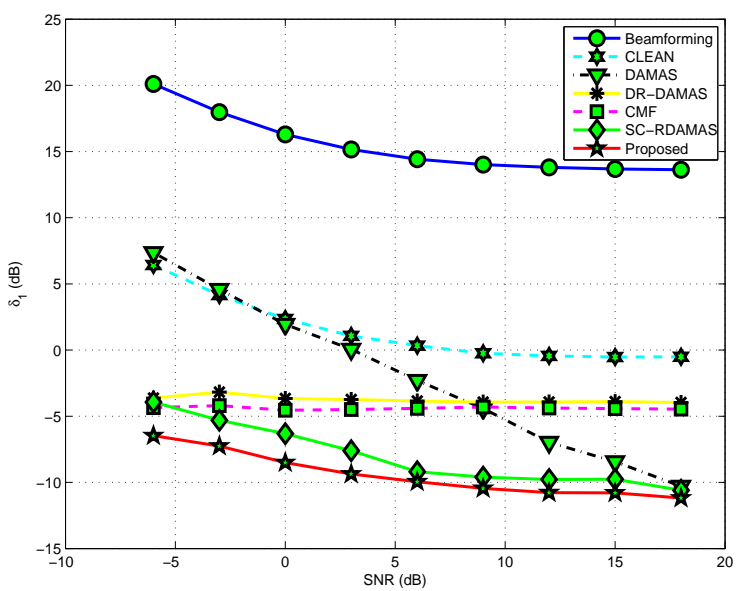

(b)

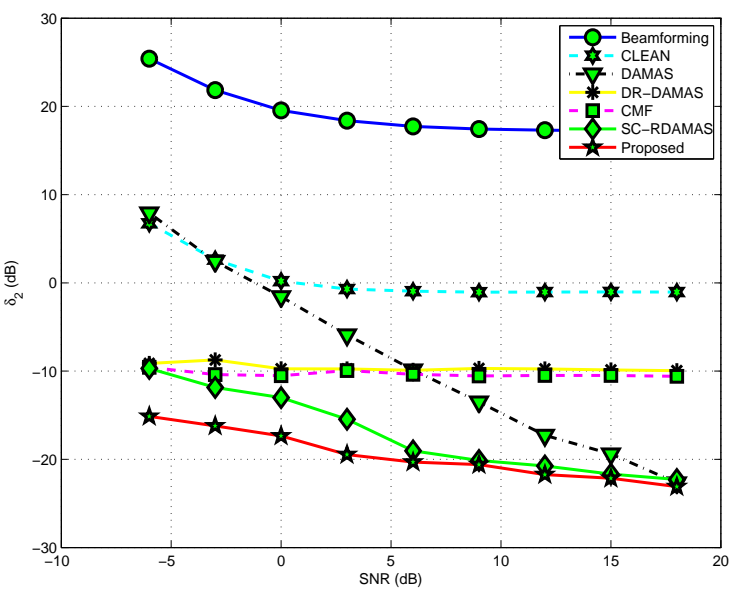

Figure 4: Performance comparison for relative errors of power image reconstruction $\delta_{1}$ and $\delta_{2}$ versus $\mathrm{SNR}[-6,18] \mathrm{dB}$ on simulations at $\mathrm{f}=2500 \mathrm{~Hz}$ (a) $\delta_{1}$ VS SNR (dB) and (b) $\delta_{2} \operatorname{VS~SNR~(dB).~}$

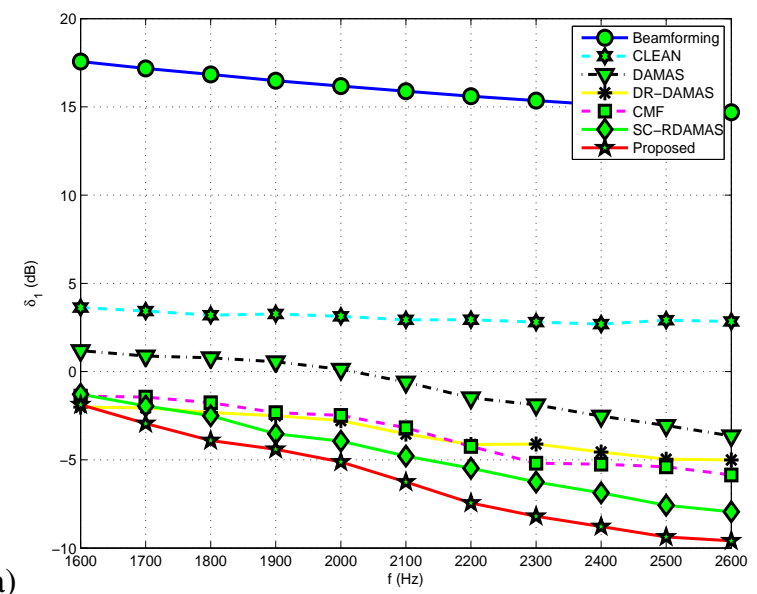

(b)

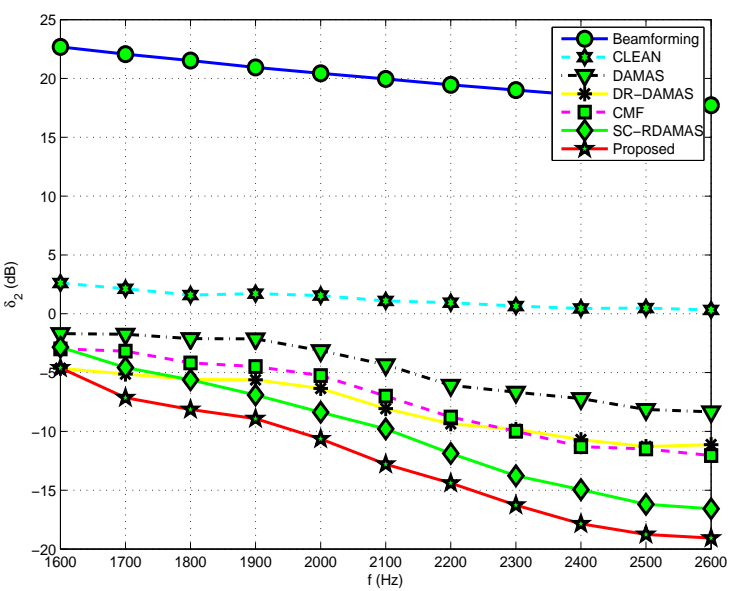

Figure 5: Performance comparison for relative errors of power image reconstruction $\delta_{1}$ and $\delta_{2}$ versus [1600,2600]Hz on simulations at $\mathrm{SNR}=3 \mathrm{~dB}$ : (a) $\delta_{1}(\mathrm{~dB}) \operatorname{VS~f}(\mathrm{Hz})$ and $(\mathrm{b}) \delta_{2}(\mathrm{~dB}) \operatorname{VS~f}(\mathrm{Hz})$.

$f \in[1600,2600] \mathrm{Hz}$. With higher frequencies, all the methods obtain better spatial resolution, indeed, our proposed method exceeds the others over the entire working frequency range.

\section{Real data of wind tunnel experiments}

The wind tunnel experiments have been carried out by Renault SAS [41] as shown in Fig.2a. This wind tunnel is designed to measure the aeroacoustic source powers and their localization on the car surface, and it can simulate the situation when Renault cars travel fast on the high-way.

\subsection{Wind tunnel experiment configurations}

Figure 2 shows the configurations of the wind tunnel S2A [41], object vehicle, NUA array and wind refraction. We suppose that all aeroacoustic sources locate on the same plane. This assumption is almost satisfied, because the curvature of the car side is relatively small compared to the distance $\mathrm{D}=4.5 \mathrm{~m}$ between the car and array plane. Since the scanning step is set by $\Delta p=5 \mathrm{~cm}$, the source plane of car side is of $1.5 \times 5 \mathrm{~m}^{2}(31 \times 101$ pixels), and we also focus on a small region of the rearview mirror: $1 \times 1.5 \mathrm{~m}^{2}(21 \times 31$ pixels $)$. On the real data, there are $\mathrm{T}=524288$ samplings 
with the sampling frequency $f_{s}=2.56 \times 10^{4} \mathrm{~Hz}$. As discussed in Section 2.2, we separate these samplings into $\mathrm{I}=204$ blocks with $\mathrm{L}=2560$ samplings in each bloc. The working frequency band is chosen as $[2400,2600] \mathrm{Hz}$, which is sensitive to human being. The image results are shown by normalized dB images with $10 \mathrm{~dB}$ span.

For the actual propagation time $\tau_{n, m}$ and distance $r_{n, m}$ in Eq.(3) in Section 2.2, we apply equivalent source to make refraction correction. As shown in Fig.2b, for sensor $m$, it seems to receive the signal from equivalent source signal $s_{n^{\prime}}$, instead of original source signal $s_{n}$, along a direct path $r_{n^{\prime}, m}$ during the same propagation time $\tau_{n^{\prime}, m}$, as if there is no wind influence in the wind tunnel. For $\tau_{-n, m}$ and $r_{-n, m}$ in Eq.(3), we use the mirror source signal $s_{-n}$ to correct the ground reflection as shown in Fig.1b. The details of the propagation corrections are discussed in Appendix $\mathrm{C}$ and Appendix D.

\subsection{Results of single frequency at $2500 \mathrm{~Hz}$}

The proposed Bayesian approach provides the following hyperparameter estimations: the variance of background noises is $\hat{\sigma}^{2}=25$; forward model uncertainty is as small as $\sigma_{\xi}^{2}=0.83$, thanks to propagation corrections in the wind tunnel, such as refraction and reflection as discussed in Appendix C and Appendix D; and the scale parameter $\gamma$ in $\mathcal{D E}(\mathbf{x})$ prior model is estimated as $\hat{\gamma}=0.33$, and this small value can insure the sparse distribution of source power with wide dynamic range, as discussed on the simulations in Section 6.

For aeroacoustic imaging on the car side in Fig.6a, the left side of Fig.6b-g illustrates the normalized estimated power images of mentioned methods. In Fig.6b, the beamforming [4] hardly obtains a clear image of source powers, it just gives a very fussy image of strong powers around the front wheel, the rearview mirror and the back wheel. In Fig.6c, DAMAS [14] successfully deconvolves the beamforming image, and discovers weak sources in the front light, front cover and side windows; however, DAMAS also gets many false targets outside the car surface. In Fig.6d, the DR-DAMAS [14] eliminates most of the false targets outside the car, but it also removes the sources on the rearview mirror and back wheel. Figure 6e shows that CLEAN [13] overcomes drawbacks of the DAMAS and DRDAMAS, but unexpected strong noises are detected on the ground; moreover, we have to set carefully the parameters in CLEAN for each experiment. In Fig.6f, the SC-RDAMAS [21] obtains a slightly better result than the CLEAN, but false alarms under the car body are still numerous. Finally, figure $6 \mathrm{~g}$ reveals that proposed Bayesian approach achieves the best performance of all, especially for noise suppression and source reconstructions on the two wheels and the rearview mirror; it removes most of the false targets under the car and on the air. The reasons of these good performances are mainly the same ones as discussed on the simulations in Section 6. The computational times of the different methods are given in Table 3. Indeed, the hyperparameter estimations increase the computational cost, but our proposed approach remains feasible to realize it.

Table 3: Computational cost for treating whole car: image $31 \times 101$ pixels, at $2500 \mathrm{~Hz}$, based on CPU:3.33GHz, '-' means unavailable.

\begin{tabular}{|c|c|c|c|c|c|c|c|}
\hline Methods & Beamforming & DAMAS & DR-DAMAS & CLEAN & SC-RDAMAS & Proposed & CMF \\
\hline Time (s) & 1 & 10 & 11 & 45 & 852 & 1012 & - \\
\hline
\end{tabular}

Based on the results of the car side, we further investigate weak sources on the rearview mirror. In Fig.7, beamforming in Fig.7a mainly demonstrates 3 groups of sources: one on the corner of the front wheel, one on left of the rearview mirror and the other on its right. DAMAS in Fig.7b improves the resolution of the beamforming, but also gets many dirty spots outside the car. In Fig.7c, most of the false alarms are removed by DR-DAMAS, but the vertical resolution is not high enough; CMF in Fig.7d obtains better results than DR-DAMAS; the SC-RDAMAS in Fig.7e achieves a result as good as CMF does; but our proposed Bayesian approach in Fig.7f achieves more expected estimations of source positions and powers around the mirror, on the front wheel and on the front corner. This result is reasonable, since these car parts are highly probable to produce aeroacoustic sources in wind tunnel.

Above all, the two experiment results agree closely with the simulation results in Fig.3 at 2500Hz. The proposed Bayesian approach is proved to achieve super-resolution, suppression of background noises and a wide dynamic range of power estimations. 
(a)

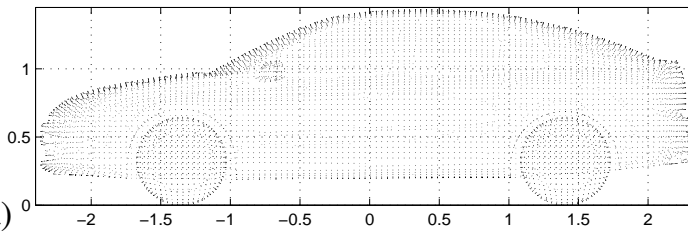

(b)

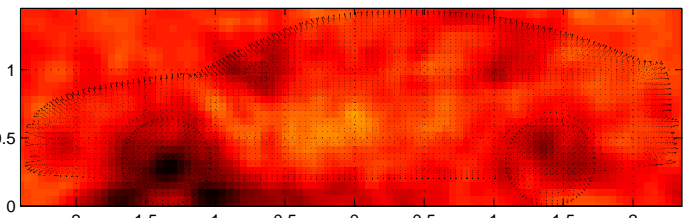

(c)

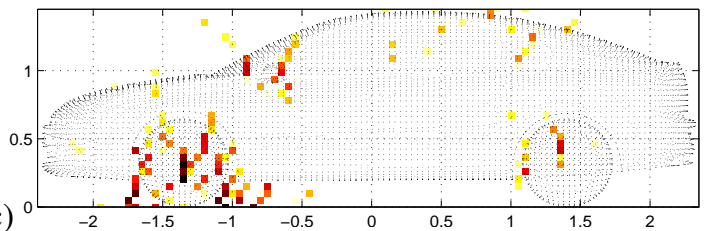

(d)
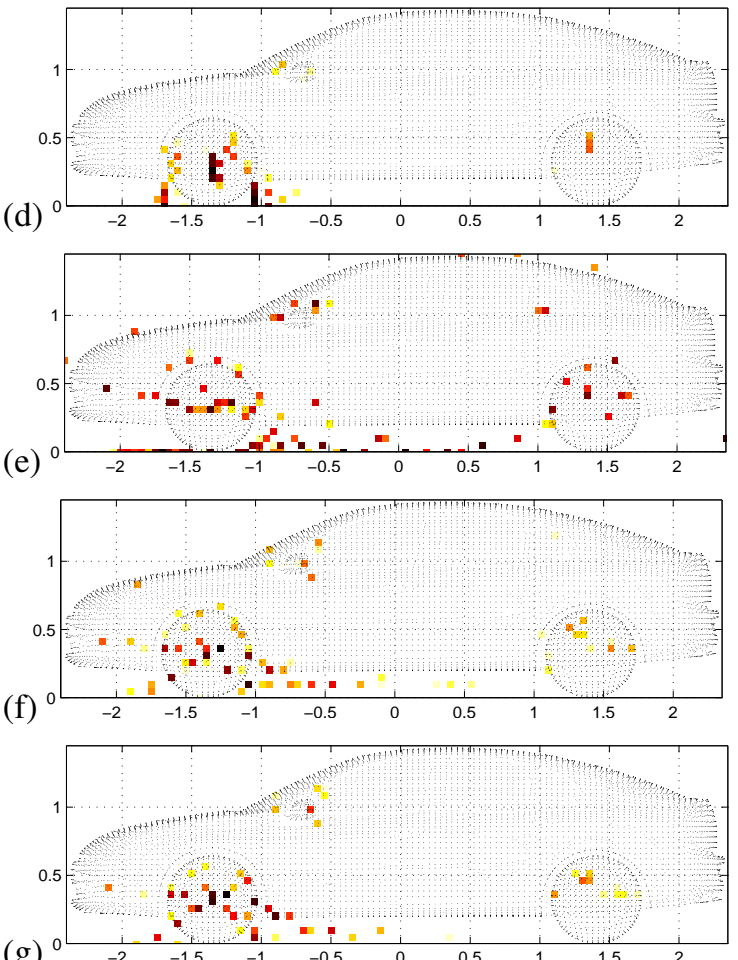

(a')
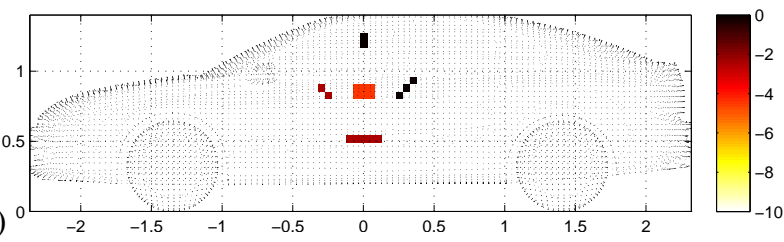

(b')

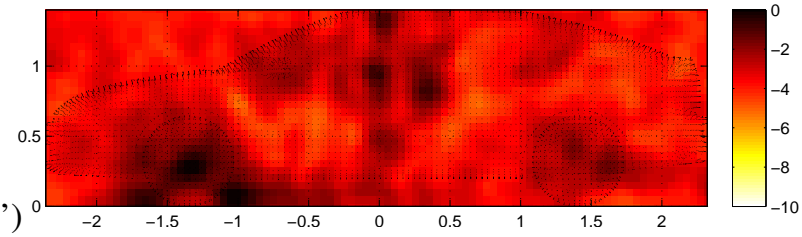

(c')

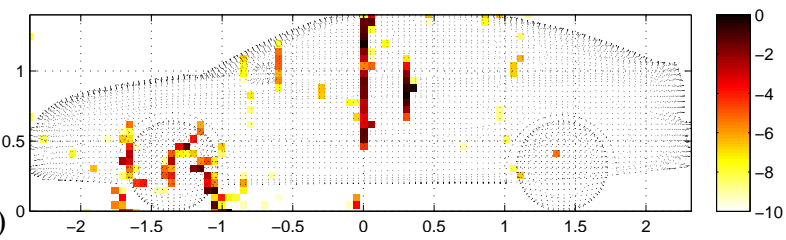

(d')

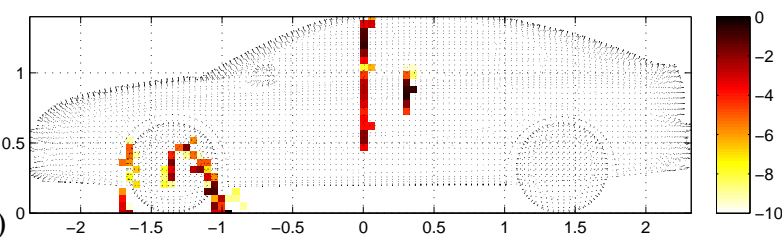

(e')
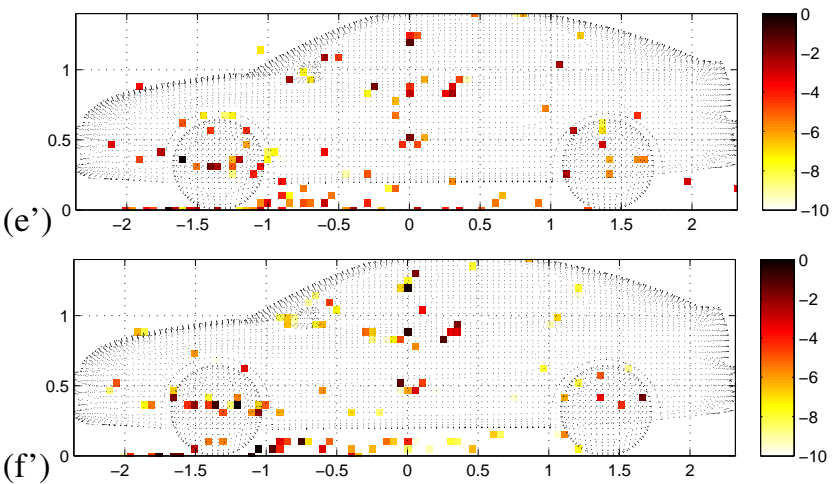

(g')

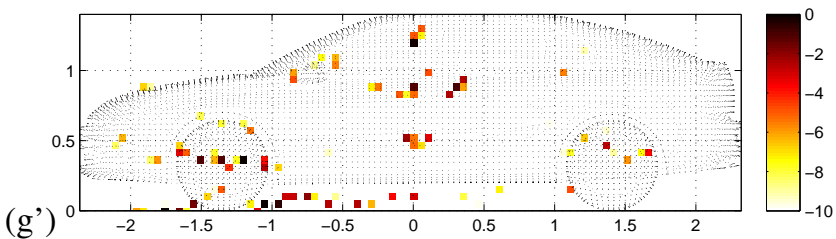

Figure 6: Aeroacoustic imaging on the vehicle side at $2500 \mathrm{~Hz}$. Left: real data (a) vehicle surface (b) Beamforming (c) DAMAS (5000i) (d) DRDAMAS (5000i) (e) CLEAN (f) SC-RDAMAS and (g) Proposed approach. Right: hybrid data (a') 5 simulated extended sources (b') Beamforming (c') DAMAS (5000i) (d') DR-DAMAS (5000i) (e') CLEAN (f') SC-RDAMAS and (g') Proposed approach.

\subsection{Results of wide-band data}

Based on the effectiveness and feasibility at single frequency, we show the performance comparisons for wideband data of $[2400,2600] \mathrm{Hz}$, as illustrated in Fig.8. Each method obtains a better result than the corresponding one at $2500 \mathrm{~Hz}$ in Fig.6. That is because the SNR is increased by averaging results over the working frequency band, and the flashing false alarms are suppressed over the wide-band average. The estimations of the DR-DAMAS in Fig.8a 

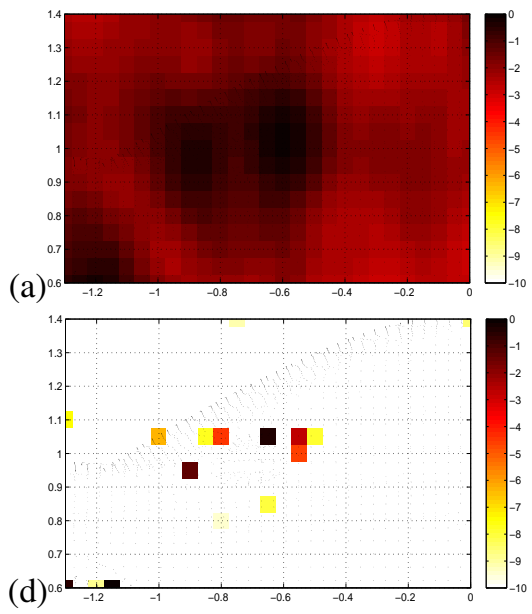

\section{(b) ${ }^{\circ}$}

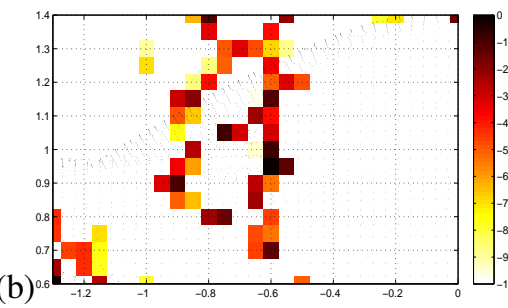

(e)

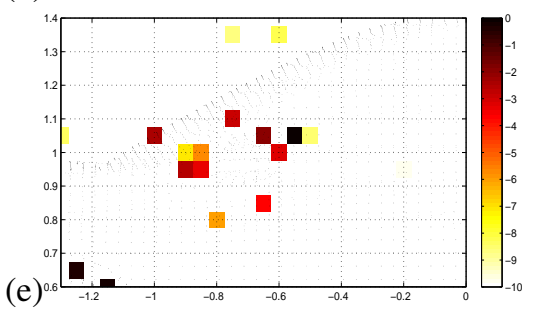

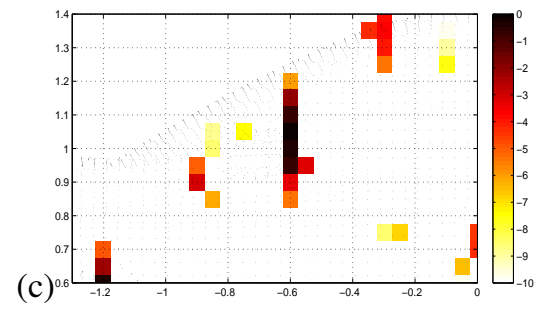

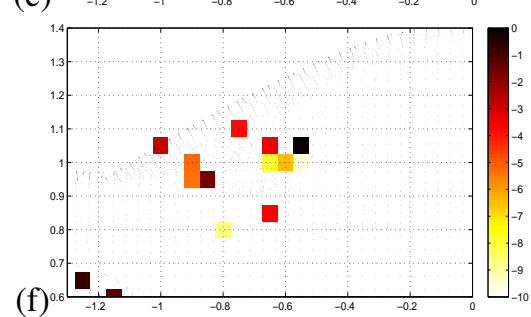

Figure 7: Aeroacoustic imaging of real data on rearview mirror part at 2500Hz: (a) Beamforming (b)DAMAS (5000i) (c) DR-DAMAS (5000i) (d) CMF (e) SC-RDAMAS and (f) Proposed

are reasonable and acceptable, but the spatial resolution are not high enough on the front wheel and rearview mirror. Fig.8b shows that the CLEAN greatly ameliorates the resolution, but it shows many unexpected spots under the car body. The SC-RDAMAS in Fig.8c has the advantages of the CLEAN, and it gets wide dynamic range of source powers around the front wheel, but it confront the same problem as CLEAN. Finally, our proposed approach in Fig.8d successfully extracts more expected source positions and powers than the above methods, for the weak ones on the mirror and back wheel, as well as the strong sources around the front wheel.

\section{Hybrid data}

Even though our proposed approach obtains good performance on real data from wind tunnel experiments, it is not sufficient to validate our proposed methods. This is because the exact sources on the vehicle generated by wind flow are not known beforehand. To further verify our methods, we propose to generate hybrid data by adding synthetic sources to the real data. In order to avoid overlapping real sources, the synthetic sources are set on the region where there are no obvious sources, as shown in Fig.6a'. We expect that our proposed approach could retrieve both the synthetic and potential real sources, respectively from the hybrid data. If these known synthetic sources are successfully detected, the proposed approach can be proved to be able to effectively recover the real sources on the vehicle surface.

\subsection{The synthetic sources model}

Based on the assumptions in Section 2.1, we suppose K' Gaussian white variables $\mathbf{w}^{\prime}(t)=\left\{w_{k}^{\prime}(t), k=1, \cdots, K^{\prime}\right\}$, with $w_{k}^{\prime}(t) \sim \mathcal{N}\left(0, \sigma_{k}^{2}\right)$, and $\sigma_{k}^{2}$ is the variance of the variable $w_{k}^{\prime}(t)$. In order to generate wide-band source signals $\left\{s_{k}^{\prime}(t), k=1, \cdots, K^{\prime}\right\}, w_{k}^{\prime}(t)$ is convoluted by the impulse response $h(t)$ (for instance Blackman filter). Thus the synthetic source signals are modeled by

$$
s_{k}^{\prime}(t)=w_{k}^{\prime}(t) * h(t), \quad k=1, \cdots, K^{\prime},
$$

where $*$ denotes convolution operation. Since the positions $\mathbf{P}^{\prime}$ of synthetic $\mathbf{s}^{\prime}$ are set to be known, the measurements $z^{\prime}$ at the sensor array can be calculated by the forward model of aeroacoustic signal propagation in Eq.(7). Then we generate the hybrid data by adding $z^{\prime}$ to the real measured data $z$.

\subsection{Results on hybrid data}

Five synthetic extended sources with different patterns are generated as seen in Fig.6a'; their powers are among $[-4.5,0] \mathrm{dB}$. The working frequency is $2500 \mathrm{~Hz}$. The right side of Fig.6b-g gives the results of mentioned methods. In 
(a)

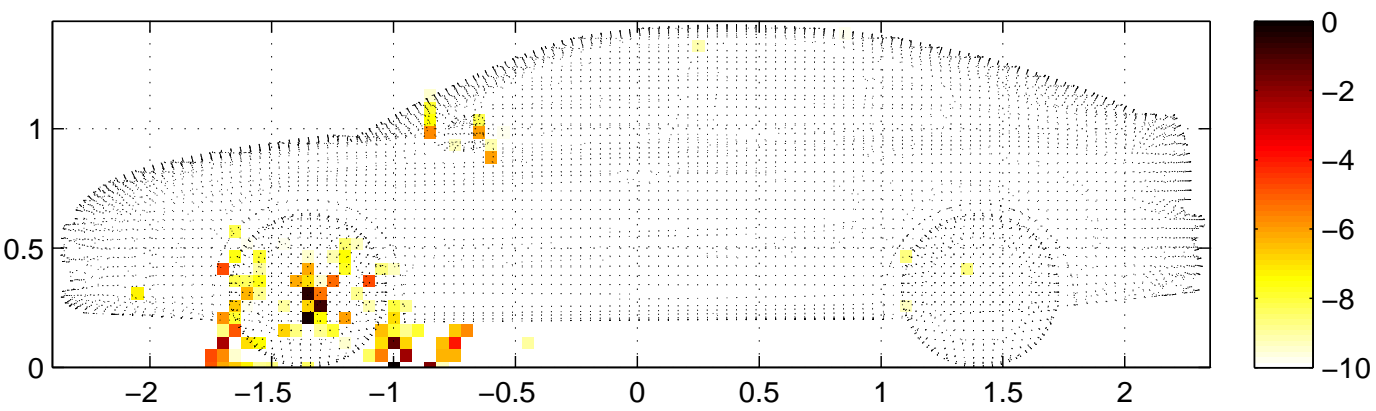

(b)
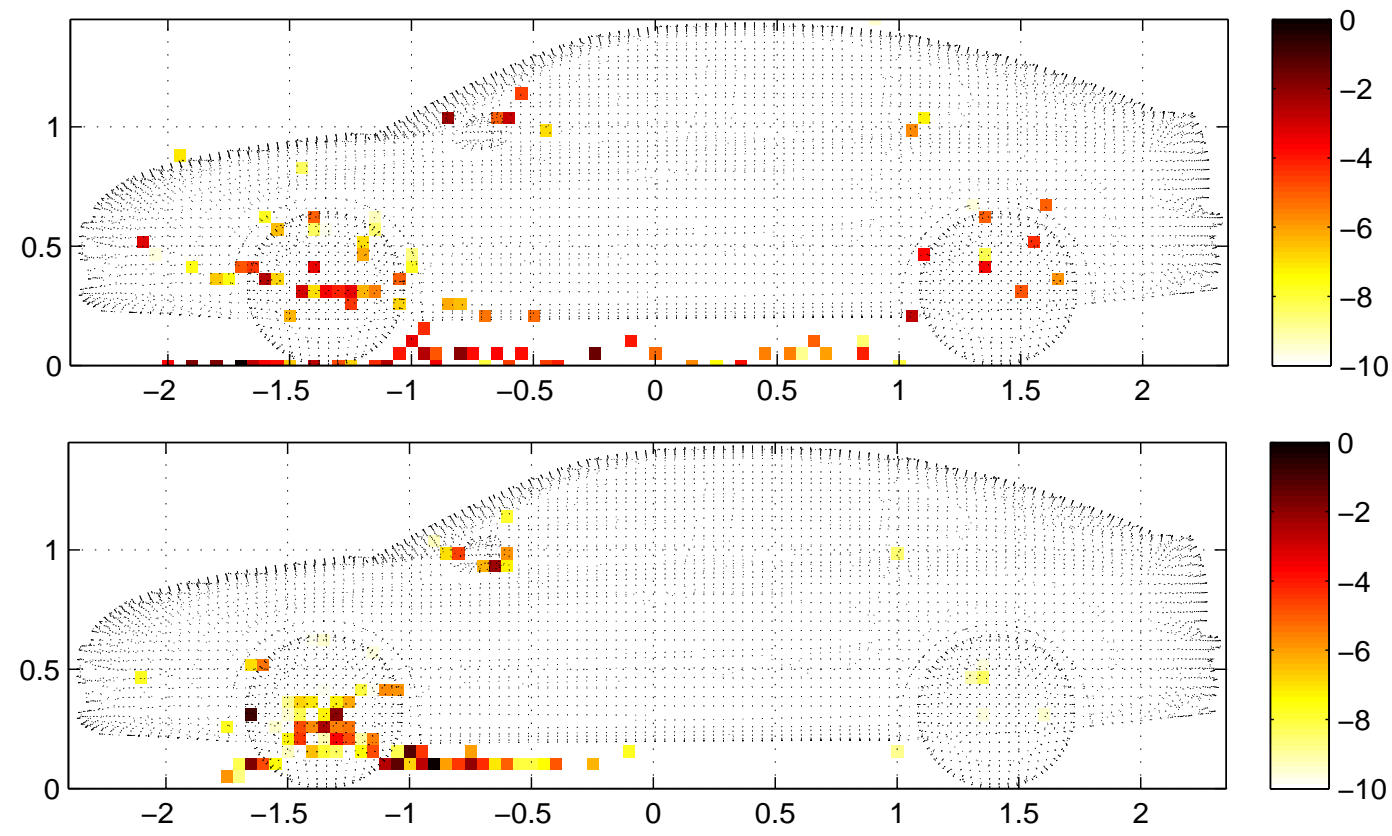

(c)

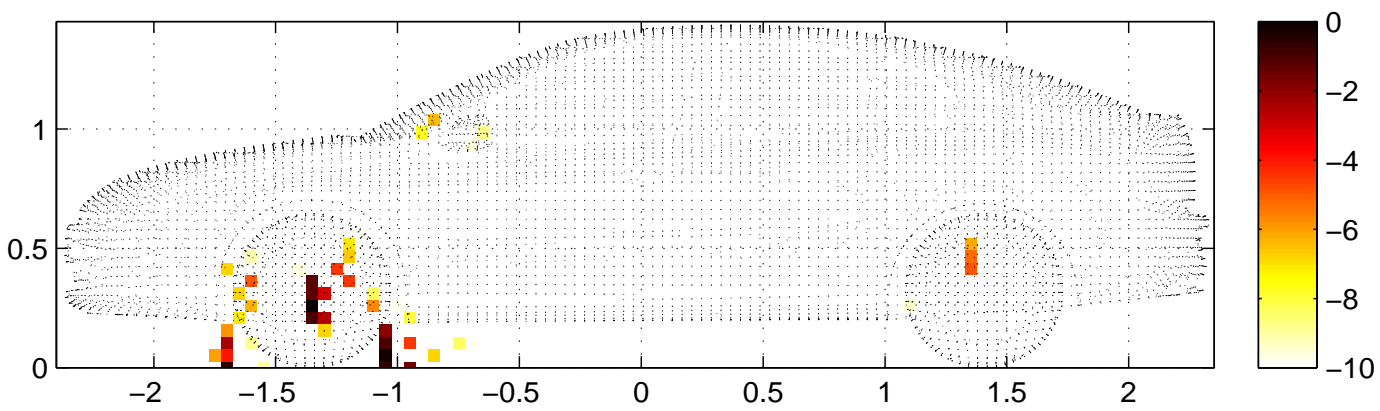

(d)

Figure 8: Wide-band data over [2400,2600]Hz: (a) DR-DAMAS (5000i) (b) CLEAN (c) SC-RDAMAS and (d) Proposed

fig.6b', the beamforming mainly shows the strong source distributions, but it reveals that there is no apparent overlaps of the measured powers between the synthetic sources and the potential ones in the real data, so that the synthetic sources are reasonably positioned beforehand; in Fig.6c' and d', both the DAMAS and DR-DAMAS fail to provide good reconstructions for the synthetic sources, due to their sensitiveness to the background noises; in Fig.6e' and f', both the CLEAN and SC-RDAMAS offer better estimations for the most of synthetic sources, and they also obtain comparable results on real data with respect to the left side of Fig.6e and f. However, CLEAN also detects many artifacts under the car due to its parameter selection, and the performance of SC-RDAMAS depends on the estimation of source number that is different between the real data and hybrid data. 
In Fig.6g', the proposed Bayesian approach successfully detects almost all the synthetic sources, and it obtains more precise estimations of their powers and patterns than the other classical methods. Meanwhile, for the other sources in the real data, the proposed approach better reconstructs both the strong sources on the two wheels and rearview mirror, and weak ones on the front cover and back window, and this result in Fig.6g' is very close to the reconstruction for the real data in Fig.6g. Moreover, proposed approach obtains simultaneously a better noise suppression and larger dynamic range of estimated powers, compared to the mentioned state-of-the-art methods.

But comparing to the result on real data in Fig.6g, we notice the hybrid data in Fig.6g', there are additional sources except the synthetic ones, such as the ones between synthetic sources and the ones under the car body. This phenomenon could be explained as follows: firstly, comparing to the beamforming powers of real data in Fig.6b, the beamforming of the hybrid data in Fig.6b' have suffered the side effect of hybrid data, since some of the sidelobes of the synthetic sources have been more or less overlapped with the mainlobes of real sources, or vice versa; so that shadow sources are inevitably detected by proposed approach, especially for the sources on the vehicle bottom; secondly, since the aeroacoustic field is linear, the beamforming powers of hybrid data can be made as the superpositions of the synthetic and real data; however, the proposed joint MAP inversion in Eq.(22) is not a linear estimator for source powers and hyperparameters, therefore small false detections would be made to some extent. But proposed joint MAP approach still achieves the expected reconstructions for the synthetic sources in hybrid data, and it is reasonable to accept the source power recovery on the real data.

\section{Conclusion}

In this paper, we have developed a robust Bayesian super-resolution approach via sparsity enforcing a priori for source localization and power reconstruction, as well as the hyperparameter estimations. Our work has been motivated to achieve the aeroacoustic imaging with super spatial resolution, large dynamic range and robustness to background noises.

The main novelties are: 1) We have firstly proposed a robust forward model of aeroacoustic power propagation by introducing the background noises at the sensor array and forward model uncertainty caused by aeroacoustic multipath propagation. The latter one is often ignored by classical methods. 2) For the inverse problem, we have explored the Bayesian inference approach via sparsity enforcing prior based on joint MAP optimization. 3) For the super resolution and wide dynamic range of source powers, we have explored the double exponential model for the sparse distribution of source powers, in which, $\beta=1$ greatly improved the spatial resolution, and proper $\gamma$ promoted the wide dynamic range of source powers. 4) For the robust imaging in strong noises, we have jointly estimated $\gamma$, noise power and forward model uncertainty, as well as the source powers via the joint MAP criterion.

The validation of proposed forward model and joint MAP inversion has been presented both on the simulated, real data and hybrid data. Firstly, various simulations have shown that our proposed approach has obtained $5 \mathrm{~cm}$ $\left(\Delta B=\arg \tan \frac{\Delta p}{D} \approx 0.6^{\circ}\right)$ super spatial resolution, $14 \mathrm{~dB}$ wide dynamic range of power estimations in $0 \mathrm{~dB}$ SNR cases for monopole and extended source imaging. Then wind tunnel experiments have demonstrated that our approach has effectively detected the expected strong sources on the front wheels and mirrors, as well as weak sources on the back wheels. Finally, hybrid data have further confirmed that proposed approach not only has well reconstructed the known synthetic sources, but also offered an expected results for real data. Moreover, proposed approach did not require the source number or SNR beforehand. However, proposed Bayesian inference increased the computational cost, but it still remained feasible to use.

For future work, we are investigating realtime implementation of proposed joint MAP approach by using the Graphical Processor Unit (GPU), and we are also exploring the hierarchical Variational Bayesian Approximation (VBA) via Student's-t distributions for acoustic imaging in colored noise.

\section{Acknowledgment}

The authors are deeply grateful to Renault SAS, especially Mr. Jean-Luc Adam for offering real data and valuable discussions on our research. We thank a lot to Dr. Nicolas GAC in L2S Lab for his valuable suggestion. 

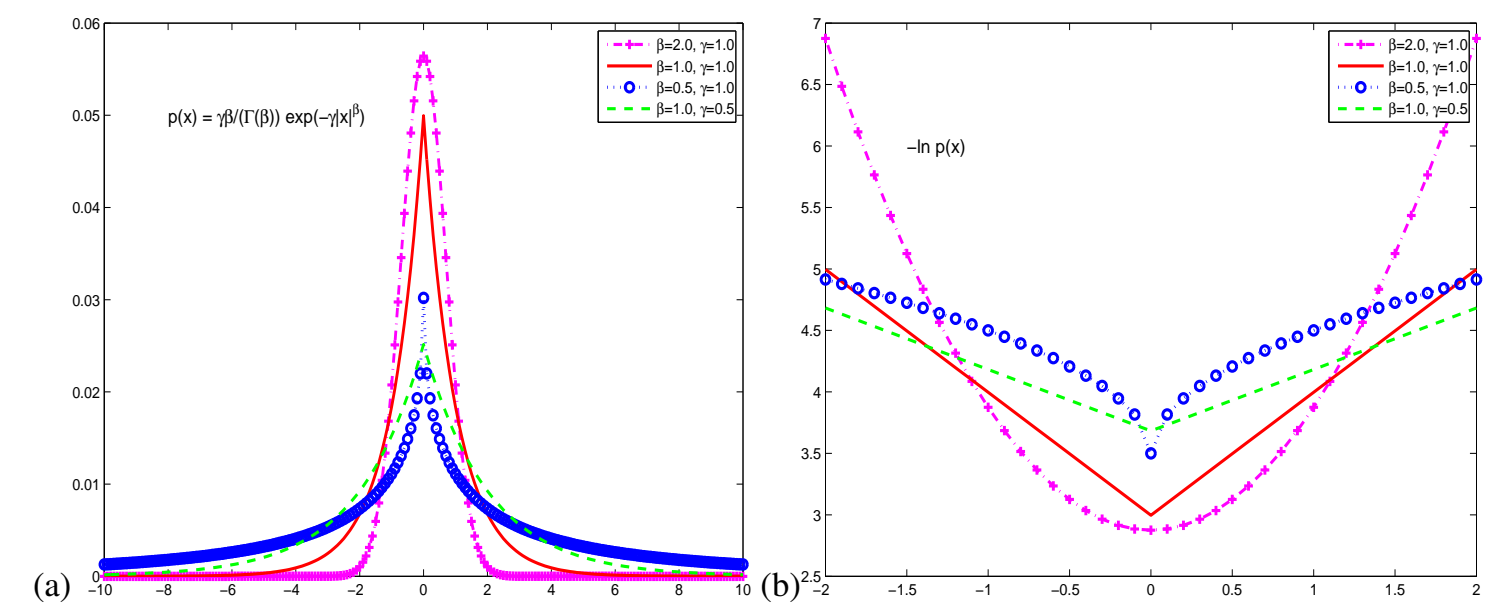

Figure A.1: Generalized Gaussian family: (a) Probability density function $\mathcal{G G}\left(x_{n}\right)$ and (b) $-\ln \left[\mathcal{G G}\left(x_{n}\right)\right]$ function.

\section{Appendix A. Sparsity enforcing a priori of source power distribution}

In Eq.(6), source signals $\mathbf{s}$ include just $\mathrm{K}$ non-zero signal $\mathbf{s}^{*}$ in the space domain, which is rather small with respect to $\mathrm{N}-\mathrm{K}$ zero values $(\mathrm{N}>>\mathrm{K})$. Therefore, the source powers $\mathbf{x}$ defined in Eq.(11) are also of the K-sparsity signals. For the PDF distribution of $\mathbf{x}$, most of the high probability values should concentrate around the original zero of $\mathbf{x}$. Meanwhile, $\mathbf{x}$ contains different items, among which, the difference between the maximal and minimal powers could be of $10 \mathrm{~dB}$ dynamic range. This large dynamic range of powers can be represented by a PDF distribution with a long heavy tail among the large values of $\mathbf{x}$. The above sparse distribution can be defined from the Generalized Gaussian $\mathcal{G} \mathcal{G}\left(x_{n}\right)$ family as discussed in $[44,45]$. Mathematically, for the uncorrelated centralized $\mathbf{x}$, the prior model based on $\mathcal{G G}\left(x_{n}\right)$ can be expressed as:

$$
p\left(\mathbf{x} \mid \boldsymbol{\theta}_{2}\right)=\prod_{n=1}^{N} \mathcal{G} \mathcal{G}\left(x_{n} \mid \gamma, \beta\right)=\left[\frac{\beta \gamma}{2 \Gamma(1 / \beta)}\right]^{N} \exp \left\{-\gamma \sum_{n=1}^{N}\left|x_{n}\right|^{\beta}\right\},
$$

where $\boldsymbol{\theta}_{2}=[\gamma, \beta]^{T}$ and

$$
\mathcal{G G}\left(x_{n} \mid \gamma, \beta\right)=\frac{\beta \gamma}{2 \Gamma(1 / \beta)} \exp \left\{-\gamma\left|x_{n}\right|^{\beta}\right\}
$$

where $\Gamma(\cdot)$ denotes the Gamma function, and shape parameter $\beta$ reflects the degree of sparsity, it controls the concentration of $p\left(\mathbf{x} \mid \boldsymbol{\theta}_{2}\right)$ at zero value; Thus the smaller $\beta$ is, the sparser $p\left(\mathbf{x} \mid \boldsymbol{\theta}_{2}\right)$ becomes. And parameter $\gamma$ reflects the inverse variance of $x_{n}$, it controls the tail of $p\left(\mathbf{x} \mid \boldsymbol{\theta}_{2}\right)$ and it affects the dynamic range of $\mathbf{x}$. Thus the smaller $\gamma$ is, the longer and heavier the tail becomes.

In the case of $\beta=2$, we get Gaussian $\mathcal{N}\left(0, \frac{1}{2 \gamma}\right)$ model:

$$
p\left(\mathbf{x} \mid \boldsymbol{\theta}_{2}\right)=\prod_{n=1}^{N} \mathcal{N}\left(x_{n} \mid 0, \frac{1}{2 \gamma}\right)=\left(\frac{\gamma}{\pi}\right)^{N / 2} \exp \left\{-\gamma\|\mathbf{x}\|_{2}^{2}\right\},
$$

where $\boldsymbol{\theta}_{2}=\gamma$, and $\gamma=\frac{1}{2 \sigma_{x}^{2}}$ reflects the inverse variance of $\mathbf{x}$, with $\sigma_{x}^{2}$ being the variance of $\mathbf{x}$.

When $\beta=1$, we get the Double Exponential $\mathcal{D E}(\mathbf{x})$ model from Generalized Gaussian $\mathcal{G} \mathcal{G}\left(x_{n}\right)$ family as follows:

$$
p\left(\mathbf{x} \mid \boldsymbol{\theta}_{2}\right)=\prod_{n=1}^{N} \mathcal{D} \mathcal{E}\left(x_{n} \mid \gamma\right)=\left(\frac{\gamma}{2}\right)^{N} \exp \left\{-\gamma\|\mathbf{x}\|_{1}\right\}
$$

where $\boldsymbol{\theta}_{2}=\gamma$. 
In Fig.A.1, 4 examples of $\mathcal{G G}\left(x_{n}\right)$ family and their $-\ln \left[\mathcal{G G}\left(x_{n}\right)\right]$ functions are illustrated. When $\gamma=1$ is fixed, the smaller $\beta$ is, the sparser $p\left(x_{n}\right)$ becomes. When $\beta=1$ is set, the smaller $\gamma$ is, the heavier tail of $p\left(x_{n}\right)$ becomes. The proper values of $\beta$ and $\gamma$ can balance the sparsity and dynamic range. For cases $0<\beta<1$, it is of great interest to enforce sparsity, but unfortunately, its $-\ln \left[\mathcal{G G}\left(x_{n}\right)\right]$ function is not convex. For the case $\beta=1$ and proper $\gamma$, the Double Exponential $\mathcal{D E}(\mathbf{x})$ model can promote sparsity and obtain wide dynamic range, moreover, its $-\ln \left[\mathcal{G} \mathcal{G}\left(x_{n}\right)\right]$ function is convex as well.

In conclusion, we select the Double Exponential $\mathcal{D} \mathcal{E}(\mathbf{x})$ model with $\beta=1$ as the sparsity enforcing a prior. Here, we have to confine the non-negative constraint on source powers $\mathbf{x} \geq 0$, since the $\mathcal{D E}(\mathbf{x})$ model is the symmetry distribution.

\section{Appendix B. Alternate estimation of hyperparameters}

For the joint MAP criterion in Eq.(22), we alternatively estimate the source powers $\mathbf{x}$ and hyperparameters as follows:

$$
\left\{\begin{array}{l}
\hat{\boldsymbol{\theta}}^{(k)}=\arg \min _{(\boldsymbol{\theta})}\left\{\mathcal{J}\left(\hat{\mathbf{x}}^{(k)}, \boldsymbol{\theta}\right)\right\} \\
\hat{\mathbf{x}}^{(k+1)}=\arg \min _{(\boldsymbol{x})}\left\{\mathcal{J}\left(\mathbf{x}, \hat{\boldsymbol{\theta}}^{(k)}\right)\right\}
\end{array},\right.
$$

where hyperparameters are $\boldsymbol{\theta}=\left[\sigma^{2}, \sigma_{\xi}^{2}, \gamma\right]^{T}$. The first iteration begins based on the simple initialization of $\mathbf{x}=0$.

Moreover, we can facilitate the estimation of $\sigma_{\xi}^{2}$ as follows:

$$
{\widehat{\sigma_{\xi}^{2}}}^{(k)}=\operatorname{tr}\{\hat{\mathbf{R}}\}-\left\|\hat{\mathbf{x}}^{(k-1)}\right\|_{1}-M{\widehat{\sigma^{2}}}^{(k-1)},
$$

where $\hat{\mathbf{R}}$ is estimated from Eq.(10), and $\operatorname{tr}(\hat{\mathbf{R}})$ represents the total power of measured signals. According to power conservation, the total measured power $\operatorname{tr}(\hat{\mathbf{R}})$ consists of the source powers $\|\mathbf{x}\|_{1}$, total noise powers $M \sigma^{2}$ at $\mathbf{M}$ sensors, and the powers of forward model uncertainty $\sigma_{\xi}^{2}$.

After hyperparameter estimation, $\hat{\mathbf{x}}^{(k)}$ are alternatively optimized by the steepest gradient algorithm based on the estimated hyperparameters $\hat{\boldsymbol{\theta}}^{(k)}=\left[{\widehat{\sigma^{2}}}^{(k)},{\widehat{\sigma^{2}}}_{\xi}^{(k)}, \hat{\gamma}^{(k)}\right]^{T}$ as follows:

$$
\hat{\mathbf{x}}^{(k)}=\hat{\mathbf{x}}^{(k-1)}+\mu \nabla J\left(\mathbf{x}, \hat{\boldsymbol{\theta}}^{(k)}\right),
$$

where $\mu$ is the step size, which could be fixed as a small value, or optimally selected as discussed in paper [46]. Since $\mathbf{x} \geq 0,\|\mathbf{x}\|_{1}=\sum_{n=1}^{N} x_{n}$, the gradient $\nabla J\left(\mathbf{x}, \hat{\boldsymbol{\theta}}^{(k)}\right)$ is obtained as:

$$
\nabla J\left(\mathbf{x}, \hat{\boldsymbol{\theta}}^{(k)}\right)=-\frac{1}{{\widehat{\sigma^{2}}}_{\xi}^{(k)}} \mathbf{C}^{H}\left(\mathbf{y}-\mathbf{C} \hat{\mathbf{x}}^{(k-1)}-{\widehat{\sigma^{2}}}^{(k)} 1_{N}\right)+\hat{\gamma}^{(k)} 1_{N} .
$$

When $\hat{\boldsymbol{\theta}}^{(k)}=\left[{\widehat{\sigma^{2}}}^{(k)},{\widehat{\sigma^{2}}}_{\xi}^{(k)}, \hat{\gamma}^{(k)}\right]^{T}$ is fixed, the joint MAP criterion in Eq. (22) is a convex quadratic minimization under linear matrix constraints. This optimization can also be solved by interior point methods using MATLAB toolbox SeMuDi [43].

\section{Appendix C. Wind flow refraction}

As shown in Fig.2b, for source n, we suppose the wind refraction takes place at point $\mathrm{p}$ on the interface between the common air and wind flow. We firstly calculate the actual propagation path $r_{n, m}$ and then obtain the actual propagation time $\tau_{n, m}$.

When the medium is uniform, $r_{n, m}$ is of a geometric distance calculated as follows:

$$
r_{n, m}=\sqrt{\left(p_{n x}-\bar{p}_{m x}\right)^{2}+\left(p_{n y}-\bar{p}_{m y}\right)^{2}+\left(p_{n z}-\bar{p}_{m z}\right)^{2}},
$$

where $\overline{\mathbf{P}}=\left[\overline{\mathbf{p}}_{1}, \cdots, \overline{\mathbf{p}}_{M}\right]^{T}$ represents $3 \mathrm{D}$ coordinates of sensor array; and sensor $\mathrm{m}$ has $\overline{\mathbf{p}}_{m}=\left[\bar{p}_{m x}, \bar{p}_{m y}, \bar{p}_{m z}\right]^{T}$. The corresponding propagation time is $\tau_{n, m}=r_{n, m} / c_{0}$, with $c_{0}$ being aeroacoustic speed in the common air. 
(a)

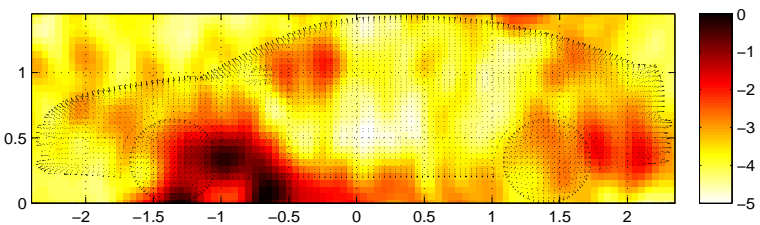

(b)

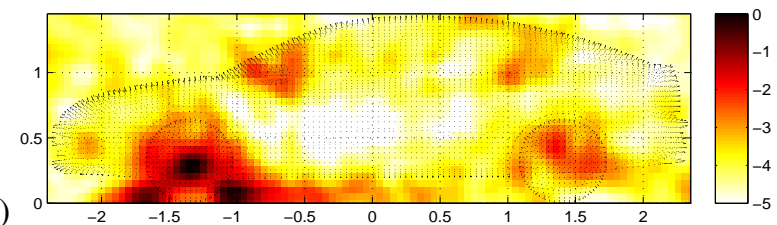

Figure C.1: Propagation corrections in wind tunnel: beamforming on the real data at $2500 \mathrm{~Hz}$ : (a) without corrections and (b) with corrections

When the medium is not uniform due to the wind flow, $r_{n, m}$ is not a geometric distance, but composed of two geometric parts as follows:

$$
r_{n, m}=d_{n, p}+d_{p, m},
$$

Where $d_{n, p}$ is the geometric distance from source $\mathrm{n}$ to refraction point $\mathrm{p}$, and $d_{p, m}$ is the one from point $\mathrm{p}$ to sensor $\mathrm{m}$.

Therefore actual $r_{n, m}$ can be calculated by knowing the position of refraction point $\mathrm{p}$.

$\tau_{n, m}$ subsequently is made up of two parts:

$$
\tau_{n, m}=\tau_{n, p}+\tau_{p, m}=d_{n, p} / c_{1}+d_{p, m} / c_{0},
$$

where $c_{1}=\left\|\vec{c}_{0}+\vec{v}\right\|$, with $\vec{v}$ being the speed vector of the wind flow. Since it is complicated to obtain $c_{1}$, we use the equivalent source $\mathrm{n}$ ' to calculate the equivalent $\tau_{n^{\prime}, p}$. We suppose that the sensor $\mathrm{m}$ seems to receive the signal from equivalent source n', instead of the source $n$, as if there were no wind influence. This means $\tau_{n, m}=\tau_{n^{\prime}, m}=d_{n^{\prime}, m} / c_{0}$. Taking the above equation into Eq.C.3, we get

$$
\tau_{n, m}=d_{n^{\prime}, p} / c_{0}+d_{p, m} / c_{0} .
$$

Therefore, actual $\tau_{n, m}$ depends on the positions of the equivalent source n' and refraction point $\mathrm{p}$.

In Fig.2.b, when the positions of sensor $\mathrm{m}\left(\overline{\mathbf{p}}_{m}=\left[\bar{p}_{m x}, \bar{p}_{m y}, \bar{p}_{m z}\right]^{T}\right)$ and source $\mathrm{n}\left(\mathbf{p}_{n}=\left[p_{n x}, p_{n y}, p_{n z}\right]^{T}\right)$ are given, we firstly locate the equivalent source n' $\left(\mathbf{p}_{n^{\prime}}=\left[p_{n^{\prime} x}, p_{n^{\prime} y}, p_{n^{\prime} z}\right]^{T}\right)$ and then determine the refraction point $\mathrm{p}\left(\mathbf{p}_{p}=\right.$ $\left.\left[p_{p x}, p_{p y}, p_{p z}\right]^{T}\right)$.

For equivalent source n', since the wind direction is parallel to the ground, the displacement exists only in horizontal direction. According to the coordinate in Fig.2.b, $\mathbf{p}_{n^{\prime}}$ can be obtained as:

$$
p_{n^{\prime} x}=p_{x n}+d_{n, n^{\prime}}, \quad p_{n^{\prime} y}=p_{n y}, \quad p_{n^{\prime} z}=p_{n z},
$$

where $d_{n^{\prime}, n}$ denotes the horizontal displacement, it can be calculated as

$$
d_{n^{\prime}, n}=d_{n^{\prime}, p} v / c_{0},
$$

where $d_{n^{\prime}, p}$ can be calculated by solving the triangles $\Delta p n n^{\prime}$ and $\Delta m n n^{\prime}$ in Fig.2b:

$$
d_{n^{\prime}, p}=\left[\frac{v}{c_{0}}\left(p_{n x}-p_{p x}\right)+\sqrt{\frac{v^{2}}{c_{0}^{2}}\left(p_{n x}-p_{p x}\right)^{2}+d_{n, p}^{2}\left(1-\frac{v^{2}}{c_{0}^{2}}\right)}\right] /\left(1-\frac{v^{2}}{c_{0}^{2}}\right),
$$

where the wind speed naturally satisfies $v<c_{0}$. And we can get $p_{p x}$ by solving the triangle $\Delta m n^{\prime} q$ on the horizontal plane in Fig.2b, and similarly $p_{p y}$ on the vertical plane. Then $\mathbf{p}_{p}$ is calculated by

$$
p_{p x}=\frac{L_{1}}{L_{1}+L_{2}} p_{n^{\prime} x}+\frac{L_{2}}{L_{1}+L_{2}} \bar{p}_{m x}, \quad p_{p y}=\frac{L_{1}}{L_{1}+L_{2}} p_{n^{\prime} y}+\frac{L_{2}}{L_{1}+L_{2}} \bar{p}_{m y}, \quad p_{p z}=\bar{p}_{m z}+L_{1},
$$

where $L_{1}$ is the distance from the sensor plane to the wind interface, and $L_{2}$ is the distance from the wind interface to the vehicle plane as shown in Fig.2b. Let $d_{m, n^{\prime}}^{\prime}$ denote the projection of $d_{m, n^{\prime}}$ on the ground. If $d_{m, n^{\prime}}^{\prime}=L_{1}+L_{2}$, then $\mathbf{p}_{p}$ can be directly calculated by Eq.(C.8). If $d_{m, n^{\prime}}^{\prime}>L_{1}+L_{2}$, we solve the the triangle $\Delta m n^{\prime} q$ based on solid geometry analysis, and finally we get the same solution as the Eq.(C.8). Above all, $\mathbf{p}_{n^{\prime}}$ is obtained by substituting Eq.(C.8) into Eq.(C.7). 
In conclusion, $r_{n, m}$ in Eq.(C.2) can be derived from the equivalent source n', and $\tau_{n, m}$ in Eq.(C.4) can be calculated from both equivalent source n' and refraction point $\mathrm{p}$. Therefore the steering vector $\mathbf{a}\left(\mathbf{p}_{n}, f_{l}\right)$ in Eq.(8) can be corrected as follow:

$$
a_{n, m}=\frac{1}{r_{n, m}} \exp \left\{-j 2 \pi f_{l} \tau_{n^{\prime}, m}\right\}+\rho \frac{1}{r_{-n, m}} \exp \left\{-j 2 \pi f_{l} \tau_{-n^{\prime}, m}\right\},
$$

where $a_{n, m} \in \mathbf{a}\left(\mathbf{p}_{n}, f_{l}\right)$ denotes the steering item from source $n$ to sensor $\mathrm{m}$, and time delay $\tau_{-n^{\prime}, m}$ of ground reflection will be discussed in Appendix D.

Our refraction correction can be extended for the other cases:

Case 1: No wind. Since $v=0$ and $L_{2}=0$, we have $d_{n^{\prime}, p}=d_{n, p}$ from Eq.(C.7), and $\mathbf{p}_{p}=\mathbf{p}_{n^{\prime}}$ from Eq.(C.8). That means that both equivalent source n' and refraction point $\mathrm{p}$ locate at the position of source $\mathrm{n}$. This conclusion meets the common sense.

Case 2: Wind everywhere. Since $v \neq 0$ and $L_{1}=0$, we have $\mathbf{p}_{p}=\overline{\mathbf{p}}_{m}$ from Eq.(C.8); it means refraction point $p$ is the same one as the sensor $m$, but horizontal displacement $d_{n^{\prime}, n}$ still takes place according to Eq.(C.7).

In Fig.C.1, we show the improvement of refraction correction. We use the real data in wind tunnel experiments, and the working frequency is $2500 \mathrm{~Hz}$. Take the beamforming method for example, it is seen that without corrections, all the sources are detected on the right side of their real positions, and there are the displacements along the wind direction; after corrections, we get the expected results.

\section{Appendix D. Ground reflection}

In Fig.1b, we show the ground reflection in wind tunnel. For the sensor m, its measured signals consists of two parts: the major one from the direct propagation, the other from the ground reflections. To correct ground reflection, here we apply the mirror source $-\mathrm{n}$ which is symmetric to the ground for source $\mathrm{n}$. For the sensor $\mathrm{m}$, it seems to receive the signal by way of the direct path $r_{-n, m}$ from the mirror source. Therefore, the steering vector $\mathbf{a}_{r}\left(\mathbf{p}_{-n}, f_{l}\right)$ of the ground reflection depends on the positions $\mathbf{p}_{-n}$ of mirror sources.

Considering $\mathbf{p}_{n}=\left[p_{n x}, p_{n y}, p_{n z}\right]^{T}$ are the coordinates of the source $\mathrm{n}$, its mirror source $\mathrm{n}$ ' has the coordinates $\mathbf{p}_{n^{\prime}}=\left[p_{n^{\prime} x}, 2 p_{n y_{0}}-p_{n y}, p_{n^{\prime} z}\right]^{T}$, where $p_{n y_{0}}$ is the vertical coordinate of the ground as shown in Fig. $1 \mathrm{~b}$. Since the original $\mathrm{O}$ of coordinate system, we have $p_{n y_{0}}=0$, thus the mirror position is $\mathbf{p}_{-n}=\left[p_{n x},-p_{n x}, p_{n x}\right]^{T}$. Therefore the steering vector of ground reflection $\mathbf{a}_{r}\left(\mathbf{p}_{-n}, f_{l}\right)$ in Eq.(8) can be calculated as similarly as the steering vector of direct path $\mathbf{a}_{d}\left(\mathbf{p}_{n}, f_{l}\right)$ in Eq.(3). Then the correspond steering vector of wind refraction $\mathbf{a}_{d}\left(\mathbf{p}_{-n^{\prime}}, f_{l}\right)$ can be corrected according to Appendix C.

Base on the analysis of ground reflection, we demonstrate the improvement of propagation corrections in Fig.C.1. As we can see, without correcting the ground reflection $(\rho=0)$, the estimated sources could be involved with the ghost sources outside and under the car; these ghosts are the influences of multipath propagation. After corrections with $\rho=0.8$, the beamforming result has much fewer ghosts in the air, on the center and rear of car body, and under the car. Furthermore, the potential strong sources are more concentrated. All the other methods are as improved as to the beamforming.

\section{References}

[1] J. Lanslots, F. Deblauwe, K. Janssens, Selecting Sound Source Localization Techniques for Industrial Applications, Sound and Vibration 44 (6) (2010) 6-10.

[2] A. B. Nagy, Aeroacoustics research in Europe: The CEAS-ASC report on 2010 highlights, Journal of Sound and Vibration 330 (21) (2011) 4955-4980.

[3] M. Magalhaes, R. Tenenbaum, Sound sources reconstruction techniques: A review of their evolution and new trends, Acta Acustica united with Acustica 90 (2) (2004) 199-220.

[4] J. Chen, K. Yao, R. Hudson, Source localization and beamforming, Signal Processing Magazine, IEEE 19 (2) (2002) 30-39.

[5] S. Lehman, A. Devaney, Transmission mode time-reversal super-resolution imaging, The Journal of the Acoustical Society of America 113 (5) (2003) 2742-2753.

[6] J. D. Maynard, E. G. Williams, Y. Lee, Nearfield acoustic holography: I. Theory of generalized holography and the development of NAH, Journal of the Acoustical Society of America 78 (4) (1985) 1395-1413.

[7] B. Van Veen, K. Buckley, Beamforming: A versatile approach to spatial filtering, ASSP Magazine, IEEE 5 (2) (1988) 4-24.

[8] A. Tarantola, Inverse problem theory and methods for model parameter estimation, Society for Industrial Mathematics, 2005. 
[9] R. O. Schmidt, Multiple emitter location and signal parameter estimation, IEEE Transactions on Antennas and Propagation 34 (1986) 276280 .

[10] E. Sarradj, A fast signal subspace approach for the determination of absolute levels from phased microphone array measurements, Journal of Sound and Vibration 329 (9) (2010) 1553-1569.

[11] M. D. Collins, W. A. Kuperman, Focalization: Environmental focusing and source localization, The Journal of the Acoustical Society of America 90 (3) (1991) 1410-1422.

[12] P. Sijtsma, Clean based on spatial source coherence, International Journal of Aeroacoustics 6 (4) (2007) $357-374$.

[13] Y. Wang, J. Li, P. Stoica, M. Sheplak, T. Nishida, Wideband RELAX and wideband CLEAN for aeroacoustic imaging, Journal of Acoustical Society of America 115 (2) (2004) 757-767.

[14] T. Brooks, W. Humphreys, A Deconvolution Approach for the Mapping of Acoustic Sources (DAMAS) determined from phased microphone arrays, Journal of Sound and Vibration 294 (4-5) (2006) 856-879.

[15] R. Dougherty, Extensions of DAMAS and Benefits and Limitations of Deconvolution in Beamforming, in: 11th AIAA/CEAS Aeroacoustics Conference, Monterey, CA, USA; 23-25 May, 2005, pp. 1-13.

[16] T. Yardibi, J. Li, P. Stoica, L. Cattafesta III, Sparsity constrained deconvolution approaches for acoustic source mapping, The Journal of the Acoustical Society of America 123(5) (May 2008) 2631-42.

[17] T. Yardibi, J. Li, P. Stoica, N. S. Zawodny, L. N. Cattafesta, A covariance fitting approach for correlated acoustic source mapping, Journal of The Acoustical Society of America 127(5) (2010) 2920-2931.

[18] D. Malioutov, M. Çetin, A. Willsky, A sparse signal reconstruction perspective for source localization with sensor arrays, IEEE Transactions on Signal Processing 53 (8) (2005) 3010-3022.

[19] N. P. Galatsanos, A. K. Katsaggelos, Methords for choosing the regularization parameters and estimating the noise variance in image restoration and thier relation, IEEE Transactions on Image Processing 1 (3) (1992) 332-336.

[20] T. Suzuki, L1 generalized inverse beam-forming algorithm resolving coherent/incoherent, distributed and multipole sources, Journal of Sound and Vibration 330 (24) (2011) 5835 - 5851

[21] N. Chu, J. Picheral, A. Mohammad-Djafari, A robust super-resolution approach with sparsity constraint for near-field wideband acoustic imaging, in: IEEE International Symposium on Signal Processing and Information Technology, Bilbao, Spain, Dec.14-17,2011, pp. 310-315.

[22] J. Antoni, A Bayesian approach to sound source reconstruction: optimal basis, regularization, and focusing, The Journal of the Acoustical Society of America 131 (2012) 2873-2890.

[23] J. Chazot, E. Zhang, J. Antoni, Acoustical and mechanical characterization of poroelastic materials using a Bayesian approach, The Journal of the Acoustical Society of America 131 (2012) 3240-3250.

[24] D. Blacodon, Spectral estimation method for noisy data using a noise reference, Applied Acoustics 72 (1) (2011) 11-21.

[25] J.-L. Adam, D. Ricot, C. Lambourg, A. Menoret, Correlated Beamforming Method for Relevant Aeroacoustic Sources Identification, in: SAE 2009 Noise and Vibration Conference and Exhibition, SAE, St. Charles, Illinois, United States, 19 May 2009, pp. 2009-01-2234.

[26] T. Brooks, W. Humphreys Jr, Extension of DAMAS Phased Array Processing for Spatial Coherence Determination (DAMAS-C), in: 12th AIAA/CEAS Aeroacoustics Conference, American Institute of Aeronautics and Astronautics, Cambridge, MA, United States, 8-10 May 2006, pp. AIAA-2006-2654.

[27] D. Blacodon, Array processing for noisy data: Application for open and closed wind tunnels, AIAA journal 49 (1) (2011) 55-66.

[28] I. Daubechies, M. Defrise, C. De Mol, An iterative thresholding algorithm for linear inverse problems with a sparsity constraint, Communications on pure and applied mathematics 57 (11) (2004) 1413-1457.

[29] R. Tibshirani, Regression shrinkage and selection via the LASSO, Journal of the Royal Statistical Society. Series B (Methodological) 58(1) (1996) 267-288.

[30] S. Chen, D. Donoho, M. Saunders, Atomic decomposition by basis pursuit, SIAM journal on scientific computing 20 (1) (1999) 33-61.

[31] E. Candès, M. Wakin, S. Boyd, Enhancing sparsity by reweighted $\ell_{1}$ minimization, Journal of Fourier Analysis and Applications 14 (5) (2008) 877-905.

[32] Y. Kim, P. Nelson, Optimal regularisation for acoustic source reconstruction by inverse methods, Journal of sound and vibration 275 (3-5) (2004) 463-487.

[33] J. Fuchs, Multipath time-delay detection and estimation, IEEE Transactions on Signal Processing 47 (1) (1999) $237-243$.

[34] F. Ronquist, J. Huelsenbeck, Mrbayes 3: Bayesian phylogenetic inference under mixed models, Bioinformatics 19 (12) (2003) $1572-1574$.

[35] A. Massa, G. Oliveri, Bayesian compressive sampling for pattern synthesis with maximally sparse non-uniform linear arrays, IEEE Transactions on Antennas and Propagation (vol.59, no.10) (Feb. 2011) 467-681.

[36] H. Jeffreys, H. Jeffreys, An invariant form for the prior probability in estimation problems, Proceedings of the Royal Society of London. Series A. Mathematical and Physical Sciences 186 (1007) (1946) 453-461.

[37] D. Tzikas, A. Likas, N. Galatsanos, The variational approximation for bayesian inference, Signal Processing Magazine, IEEE 25 (6) (2008) $131-146$.

[38] A. Gelfand, A. Smith, T. Lee, Bayesian analysis of constrained parameter and truncated data problems using Gibbs sampling, Journal of the American Statistical Association 87 (418) (1992) 523-532.

[39] O. Siohan, C. Chesta, C. Lee, Joint maximum a posteriori adaptation of transformation and HMM parameters, IEEE Transactions on Speech and Audio Processing 9 (4) (2001) 417-428.

[40] H. Anderson, M. Gupta, Joint deconvolution and classification with applications to passive acoustic underwater multipath, The Journal of the Acoustical Society of America 124 (2008) 2973

[41] A. Menoret, N. Gorilliot, J.-L. Adam, Acoustic imaging in wind tunnel S2A, in: 10th Acoustics conference (ACOUSTICS2010), Lyon, France, 2010.

[42] C. E. Kassis, J. Picheral, C. Mokbel, Advantages of nonuniform arrays using root-music, Signal Processing 90(2) (2010) 689-695.

[43] J. Sturm, Using sedumi 1.02, a matlab toolbox for optimization over symmetric cones, Optimization methods and software 11 (1-4) (1999) $625-653$.

[44] C. Bouman, K. Sauer, A generalized gaussian image model for edge-preserving map estimation, Image Processing, IEEE Transactions on 
2 (3) (1993) 296-310.

[45] A. Mohammad-Djafari, Bayesian approach with prior models which enforce sparsity in signal and image processing, EURASIP Journal on Advances in Signal Processing 2012 (1) (2012) 52.

[46] K. Kiwiel, Convergence and efficiency of subgradient methods for quasiconvex minimization, Mathematical programming 90 (1) (2001) $1-25$. 